\title{
RNAi-based biosynthetic pathway screens to identify in vivo functions of non-nucleic-acid-based metabolites such as lipids
}

\author{
Hongjie Zhang ${ }^{1,2}$, Nessy Abraham ${ }^{1,2}$, Liakot A. Khan ${ }^{1}$, and Verena Gobel ${ }^{1}$ \\ ${ }^{1}$ Mucosol Immunology and Biology Research Center, Department of Pediatrics, Massachusetts \\ General Hospital and Harvard Medical School, Boston, MA 02114, USA
}

\begin{abstract}
The field of metabolomics continues to catalog new compounds, but their functional analysis remains technically challenging, and roles beyond metabolism are largely unknown. Unbiased genetic/RNAi screens are powerful tools to identify the in vivo functions of protein-encoding genes, but not of non-proteinaceous compounds such as lipids. They can, however, identify the biosynthetic enzymes - of these compounds- findings that are usually dismissed, as these typically synthesize multiple products. Here, we provide a method using follow-on biosynthetic-pathway screens to identify the endpoint biosynthetic enzyme and thus the compound through which they act. The approach is based on the principle that all subsequently identified downstream biosynthetic enzymes contribute to the synthesis of at least this one end product. We describe how to: systematically target lipid biosynthetic pathways; optimize targeting conditions; take advantage of pathway branchpoints; and validate results by genetic assays and biochemical analyses. This approach extends the power of unbiased genetic/RNAi screens to identify in vivo functions of nonnucleic-acid-based metabolites beyond their metabolic roles.
\end{abstract}

\section{Introduction}

The role of metabolites in biological functions other than metabolism is likely to be considerably underappreciated given the technical difficulties of functionally characterizing these compounds. For instance, lipids are major components of membranes, but their role in morphogenesis is largely unknown ${ }^{1,2}$. A key technical problem is the inaccessibility of nontemplate-derived compounds to specific in vivo targeting techniques such as gene knockouts or RNAi knockdowns that constitute the basis for the functional analysis of proteins. Although mass spectrometry (MS)- and NMR-based metabolomic approaches offer systems-level annotation and characterization of metabolites and their specific moieties, they cannot identify a causal relationship between a specific metabolite and its cellular function $^{3-5}$. Imaging techniques are not yet of high spatial resolution (e.g. the emerging MSbased techniques); they lack molecular specificity or interfere with function (e.g. of lipids on membranes) ${ }^{6}$; and the large panel of antibodies available for the detection of proteins, does

Correspondence should be addressed to V.G. (vgobel@mgh.harvard.edu).

${ }^{2}$ Present addresses: Faculty of Health Sciences, University of Macau, China (H.Z); University of Alabama School of Medicine, Huntsville, AL 35801, USA (N.A.).

Note: Any supplementary Information and Source Data files are available in the online version of the paper 
not exist for lipids. Here we co-opt the successful strategy of nucleic acid targeting to investigate non-nucleic acid-derived metabolites such as lipids.

In a recent genome-wide RNAi tubulogenesis screen we identified a number of lipidbiosynthetic enzymes whose knockdowns generated the same specific polarity phenotype ${ }^{7}$. To find a putative common lipid compound through which these enzymes might act, we designed biosynthetic pathway screens and successfully identified ceramide glucosyltransferases (CGTs) as the endpoint biosynthetic enzymes, thereby revealing their single product glucosylceramide (GlcCer; the glycosphingolipid [GSL] backbone) as the common polarity-affecting lipid. Distinct biochemical functions of other identified enzymes, complemented by MS, characterized the compound being sought as a hydroxylated GlcCer (GlcCer-OH) with a C17-monomethylbranched-chain fatty acid (mmBCFA) and a saturated long-chain fatty acid (LCFA) of likely C22 length. No other existing technique could have identified this specific complex lipid's function by selectively removing it, nor would its biosynthetic enzymes have been identified in a forward mutagenesis/RNAi screen, as it required a double paralog knockdown. This analysis demonstrates that this biosynthetic pathway screen approach can be used to query, in an unbiased manner, the in vivo cell biological functions of non-DNA-encoded metabolites beyond their roles as biosynthetic substrates or metabolic products.

The approach consists of a stepwise, systematic, although not comprehensive, targeting of all the molecules of the biosynthetic pathway of which the originally identified enzyme is a part. The same genetic/RNAi technique is used to search for a copy of the original phenotype with the goal of identifying the endpoint biosynthetic enzyme in the pathway, and thereby the underlying product species, and perhaps the exact chemical characteristics of the compound. The approach (1) eliminates potential bias by starting the inquiry with the analysis of the function (phenotype) rather than the molecule of interest (a 'forward' rather than a 'reverse' molecular analysis); (2) bypasses the problem of interconversion and compensation that creates a particular difficulty for the functional analysis of lipids ${ }^{8,9}$ and (3) overcomes the obstacle that one enzyme synthesizes many products and affects different functions. This method can be used not only to functionally characterize metabolic products of biosynthetic genes that have already been identified in time-consuming genome-wide RNAi screens (typically set aside as 'housekeeping' genes) but also to design 'lipid/ metabolite' screens upfront by constructing RNAi libraries from their biosynthetic genes for the functional characterization of their products.

The strength of the approach lies in its potential to identify the mostly unknown nonmetabolic (rather than the generally well-known metabolic) cellular roles of the multitude of specific metabolic compounds (rather than their enzymes). However, it also characterizes the biosynthetic functions of the enzymes in vivo, which, in turn, may not only identify novel biosynthetic functions but also expand or revise our knowledge about the biosynthetic pathways themselves, often only established in vitro or in a restricted number of species. Moreover, in combination with techniques such as liquid chromatography (LC)-MS and gas chromatography (GC)-MS, that determine compound structure, this approach will also identify species-specific different or altogether novel compounds, together with their functions. To detect such features and incorporate them into the analysis, biochemical 
procedures are integrated into this overall genetic screen approach that otherwise relies on the particularly high conservation of metabolic enzymes between different species.

\section{Applications of the protocol}

Here, we provide a detailed technical protocol on how to design and carry out such a biosynthetic pathway screen, combined with genetic and biochemical analyses. We demonstrate the approach with lipids, specifically, membrane lipids, and illustrate it with our prior RNAi feeding screen on epithelial polarity in C. elegans (see refs. ${ }^{10-17}$ and http:// www.wormbase.org for the expanding field of lipid biology and metabolomics in $C$. elegans). The concept of the approach should, however, be equally effective in other genetic model organisms (limited only by their accessibility to specific targeting procedures), and it should serve to identify the functions of other categories of lipids or other non-templatederived compounds, such as, for instance, sugars. With the advent of scalable knockout/ knockdown techniques (RNAi and more recently clustered regularly interspaced short palindromic repeats (CRISPR) technology, forward screens, the method proposed here, can also be performed in mammalian cell lines. On the other hand, the use of live genetic screens in model organisms, not feasible in humans, is particularly well suited to the functional dissection of biosynthetic pathways and can serve as a direct means to investigate the nonmetabolic functions of their well-conserved human orthologs. As such screens start from the phenotype, not the metabolite, they are not limited by species specificities of this metabolite, such as for instance fatty acid (FA) chain length; but, when combined with biochemical analyses, have the potential to identify the exact chemical composition of the causative metabolic end product.

\section{Strengths and limitations of this method's ability to identify the causative end product}

The biosynthetic-pathway screen approach relies on the copy of a signature phenotype that is generated by interference with one or several of multiple biosynthetic enzymes in a linear pathway (Figs. 1 and 2). The depletion of any intermediate (via its biosynthetic enzyme) in a specific pathway should (in principle) produce this phenotype, but inadvertently such screens also test conditions in which these same intermediates are increased, e.g., as accumulating substrates upstream of the catabolizing enzymes that are being tested via their depletion. Thus, seemingly paradoxically, the same phenotype may be present in animals that are depleted of a specific intermediate (by interference with its upstream biosynthetic enzyme) and in animals in which this same intermediate accumulates (secondary to interference with its downstream biosynthetic enzyme). However, in the latter scenario (accumulation of the intermediate), the phenotype is caused by the depletion of further downstream products, not by accumulation of this intermediate. This property of pathway screens, the simultaneous testing of intermediates as substrates and products, constitutes this approach's strength in uniquely addressing an inherent problem of metabolic analysis: the circularity of biosynthetic pathways and the possiblity of compound interconnectivity (Figs. 1 and 2) ${ }^{18}$ It provides exceptionally strong evidence to demonstrate that it is the endpoint biosynthetic end product, not its upstream intermediates, that mediates the function under study. For instance, the same polarity defects were present in animals with loss and gain of ceramide (Cer) in our lipid bisoynthetic pathway analysis (via depletion of Cer's upstream 
and downstream enzymes, respectively), suggesting that the mediating lipid compound sought was a further downstream product, not Cer itself (in this case GlcCer) ${ }^{7}$.

Although the biosynthetic pathway screen approach addresses the problem of interconversion, it may not always solve it, nor can it overcome the problem of biosynthesis compartimentalization to different subcellular membrane systems. For instance, only the additional absence of the phenotype in a null mutant of its catabolizing enzymes would unequivocally exclude the possible contribution of the next step metabolite to the function that is being examined. This becomes an obvious challenge in cases in which a non-endpoint intermediate is suspected to execute the function of interest, which is indicated, for instance, by the absence of phenotypes in animals depleted of all its downstream metabolizing enzymes. Such a scenario may require the construction of null mutants of all immediate downstream pathway components, which may consist of several enzymes, including their paralogs. Moreover, in this particular case, strict validation of the intermediate by compound rescue of the phenotype would require the construction of a double (multiple) mutant of the immediate upstream (synthesizing) and downstream (catabolizing) enzymes. It is therefore particularly difficult to characterize functions of pathway hub compounds, such as Cer, which is a substrate of three and a product of four different metabolic reactions in $C$. elegans, most of which are catalyzed by several paralogous enzymes (Fig.2). Finally, the compartimentalization of lipid biosynthesis to different endomembrane and plasma membrane domains may cause restrictions to these biochemical rules, the functional consequences of which cannot currently be predicted. However, none of these problems can be addressed at present by any other available in vivo technique.

\section{Experimental design}

Procedures are provided for the scenario that one or several upstream FA biosynthetic enzyme genes have been identified in an unbiased screen as being required for a function of interest on the basis of their C. elegans RNAi phenotypes. As shown in Figure 3, a broad initial pathway screen (I; Steps 10-27) is designed to identify the underlying metabolite category/class and pathway branch; this is followed by an in-depth second screen (II; Steps 34 and 35) of this branch, aiming to identify the specific end product, followed (optionally) by a screen for derivatives of this end product (III; Steps 59-61), with the potential to identify its exact chemical characteristics. The direct identification of enzymes synthesizing complex lipids that are located further downstream in the pathway may make the broad screen (I) dispensible (see Supplementary Fig. 1 for complex lipid classes and their chemical compositions). The ancillary genetic and biochemical analyses serve to validate results, to connect results between different screen tiers and to exclude pathway branches, thereby reducing the number of enzymes requiring screening.

\section{Criteria for starting a biosynthetic pathway screen (Pre-screen confirmation and analysis: Steps 1-9)}

In principle, the identification of only one biosynthetic enzyme in a genetic/RNAi screen merits searching for the specific product that mediates its function. When the identified enzyme generates only one product, no further screening is necessary. More commonly, however, it generates multiple intermediates that require further analysis. The identification 
of more than one biosynthetic enzyme whose losses produce the same phenotype is particularly reassuring; and the identities of these enzymes may already be informative for the screen design. Biosynthetic pathway screens are particularly useful for the analysis of 'upstream' (in relation to their endproduct) biosynthetic enzymes that contribute to the generation of multiple products and thus reveal little information with regard to the identity of the product mediating the investigated function. In our polarity screen, the losses of four lipid biosynthetic enzymes were found to generate the same specific phenotype, which strongly suggested they function through a shared lipid compound ${ }^{7}$. Three of them were common fatty-acid (FA)-biosynthetic enzymes, one of which was the first enzyme for all FA biosynthesis ( pod-2/acetyl-CoA carboxylase(ACC)), itself giving no clue as to the nature of this compound (Fig. 1).

Several points should be checked before embarking on follow-on pathway screens (Fig. 3). First, the specificity and robustness of the phenotype is essential for the analysis that relies on its tracking. Lethality or slow growth, for instance, may be induced by interference with any of various lipids and are thus not useful for tracking a single pathway; nor is a phenotype with low penetrance or expressivity. Second, enzyme knockdowns should be validated in a corresponding germline mutant that is expected to display the same or a related phenotype, particularly if only one enzyme was identified. Third, it is useful to exclude upfront the possibility that the phenotype results not from the loss of a product downstream of the missing enzyme but rather from the accumulation of a substrate upstream of the missing enzyme, a possible alternate cause (various lipid storage diseases, for instance, are caused by toxic substrate accumulation $)^{19}$. The identification of more than one biosynthetic enzyme, or of first-step biosynthetic enzymes, may already suggest that their (common) loss-of-function phenotype is not caused by an accumulating substrate. This can be ascertained by rescuing the phenotype with exogenously supplemented lipids, provided that the identified enzyme synthesizes a product that can be substituted (an expected scenario, as pathway screens are likely to be used to analyze early acting, upstream biosynthetic enzymes that generate simple FAs). Compound rescue simultaneously rules out the third, less likely, cause of a phenotype: a dual-specific function of the enzyme that is unrelated to its biosynthetic product.

\section{Broad initial pathway screening with a focus on simple metabolites: identifying the metabolite class (Screen I: Steps 10-27)}

Lipid biosynthetic pathways can be divided into three parts that build on each other (Fig.1): (1) short- and medium chain FA biosynthesis (upstream; top); (2) LCFA biosynthesis (middle); (3) complex lipid biosynthesis (downstream; bottom). Synthesis of both short- and medium-chain FAs and LCFAs involves four-step elongation cycles (condensation, reduction, dehydration and reduction, depicted for LCFA synthesis), with malonyl-CoA, which is synthesized by ACC, as chain extender. Medium-chain FAs and LCFAs can be further modified, for example, through desaturation by desaturases (right) and/or hydroxylation by hydroxylases (not shown). They then serve as precursors for complex lipids (bottom). 
Not infrequently the loss of first-step, often rate-limiting, biosynthetic enzymes generate detectable phenotypes, which implicate the entire field of possible downstream products as candidate mediating compounds. The first-tier follow-on pathway RNAi screen searching for phenocopy should assemble an RNAi library that targets genes involved in a wide range of lipid-related processes, including synthesis, transport and regulation, with an emphasis on the (upstream) basic machinery of FA metabolism (the size of libraries typically ranges from $\sim 100$ to 300 molecules, scalable depending on ease of phenotypic evaluation ${ }^{7}$; Table 1 shows a collection of critical $C$. elegans FA biosynthetic enzymes). This analysis is targeted towards the identification of clues, and it should thus be broad rather than comprehensive.

Any positive hit will be both confirmatory with regard to the initially identified enzyme and newly informative with regard to the further screen design. For example, the identification of an elongase in a first-level follow-on pathway screen for an initial identification of ACC/ pod-2 (Fig. 1): (1) supports the functioning of ACC/pod-2 via a downstream lipid compound; (2) identifies an LCFA component of this lipid compound and (3) may reveal depending on what elongase was identified - whether this LCFA is branched or straight. Of nine $C$. elegans elongases, ELO-5 and ELO-6 have been characterized as producing branched-chain LCFAs (C15ISO and C17ISO) ${ }^{12}$, and thus their identification would reveal the presence of a mmBCFA in the compound in question (Fig. 1) ${ }^{12,20 .}$

\section{Confirming the involvement of a specific pathway branch (Post screen I confirmation and analysis: Steps 28-30)}

It is not necessary to probe every enzyme of the pathway, but predictions can be tested for further confirmation: for example, our first-level follow-on lipid biosynthetic pathway screens identified two polarity-affecting enzymes catalyzing successive steps of fatty acid elongation: the elongase ELO-3 and the 3-ketoacyl-CoA-reductase/KAR LET-767. Probing the enzyme catalyzing the reaction immediately downstream of KAR/LET-767 additionally identified the 3-hydroxyacyl-CoA dehydratase T15B7.2, thereby confirming pathway linearity (Fig. 1 and 3$)^{7}$.

Identifying the phenotype of interest in animals depleted of a first step branch-specific enzyme will immediately direct follow-on screens to the corresponding branch. To increase confidence in the specific branch's involvement, findings can be validated by the analysis of the corresponding germline mutants and/or by rescuing the phenotype via a downstream lipid compound. As complex lipids - in contrast to FAs - may not be easily available for rescue, enzyme inhibitors can be used to evaluate whether they copy the phenotype of interest. Many small-molecule inhibitors have been developed to modify lipid biosynthetic enzyme activities $^{21-25}$ (Tables 1, 2). For example, inhibitors of serine palmitoyltransferase include sphingofungins, lipoxamycin and myriocin. In $C$. elegans, they can generally be delivered by feeding. Inhibitors are often competitive substrates, and thus the same precautions apply for their supplementation as for lipid feeding (steps 5-9). Their efficiency at inhibition in vivo varies, thus, although phenocopy will be confirmatory, a negative result will not rule out the targeted enzyme. 


\section{Taking advantage of intermediate modifications and pathway branchpoints (Post-screen I confirmation and analysis: Steps 31 and 32)}

Negative RNAi results, although informative, cannot by themselves exclude an enzyme as a pathway component. They could be caused by a number of reasons, including clone- or gene-specific RNAi resistance and redundancies, the latter being a typical scenario for biosynthetic enzyme genes, which often have paralogs (Tables 1 and 2). Although the screen design is guided by positive results, negative results can be used for branch exclusion (Fig. 3). In this case, further confirmation is nessessary, with rigorous proof attainable only with germline null mutants; thus, it may require the construction of double, triple or even quadruple mutants. It is time-efficient to construct or obtain such mutants (many already available) if, with their help, large numbers of compounds can be excluded. This will be the case if the identified enzymes operate upstream in the pathway, thereby contributing to the synthesis of multiple compounds. For instance, the exclusion of desaturation has the potential to rule out a large proportion of simple, as well as complex, lipids, inclusive of all glycerolipids and phospholipids (Fig. 1). C. elegans contains three delta-9 desaturases, which are enzymes that carry out the first desaturation of 16:0 and 18:0 to $16: 1 \mathrm{n} 7$ and 18:1n9 and thus generate mono unsaturated FAs with fat-5 encoding a palmitoyl-CoA desaturase and fat- 6 and fat-7 encoding stearoyl-CoA desaturases (Fig. 1). Therefore, if the fat-6(tm331); fat-5(tm420) fat-7(wa36) triple mutant, a severe loss-of-function mutant ${ }^{13}$, lacks the phenotype of interest, this will suggest that unsaturated FA-containing lipids do not mediate the function of the initially identified biosynthetic enzyme (although it will not, of course, exclude their function in other aspects of the same process). FAT-2, the only delta-12 desaturase in $C$. elegans, catalyzes the first reaction of polyunsaturated FA (PUFA) biosynthesis ${ }^{10}$. Lack of the phenotype of interest in the nonredundant strong loss-of-function allele fat-2(wa17), which was demonstrated to be completely devoid of normal PUFAs ${ }^{13}$, would exclude PUFA-containing lipids, for instance eicosanoids. In our study on polarity, we could exclude phosphoinositides - which were prime candidates given their welldescribed roles in polarity - on the basis of absence of the polarity phenotype in fat-2(wa17) ${ }^{7}$. In principle, the same concept could be applied for any other type of compound modification (e.g., hydroxylation).

Pathway branchpoints can furthermore be exploited in this way to exclude entire branches. The enzymes that catalyze the first committed (and rate-limiting) steps of sphingolipid (SL) and glycerolipid/glycerophospholipid biosynthesis (Fig. 2a,b), respectively, are serine palmitoyltransferase and glycerol-3-phosphate acyltransferase or dihydroxyacetone phoshate acyltransferase. $C$. elegans contains three serine palmitoyltransferases (SPTL-1, 2 and 3) and three glycerol-3-phosphate acyltransferases (ACL-4, 5 and 6). The latter have been shown to acetylize both, dihydroxyacetone phosphate and glycero-3-phosphate, which makes them sufficient targets for branch exclusion (Figure 2b, top) ${ }^{26}$. The absence of the phenotype of interest in triple sptl-1, 2, 3 or acl-4, 5, 6 null mutants would indicate that the corresponding branches (SLs and glycerolipids/glycerophospholipids, respectively) are not involved in the investigated function. Before attempting to construct such triple-null mutants, it should be considered that they might be sterile or early lethal, limiting their usefulness for the tracking of phenotypes occuring at later stages. Moreover, lethal mutants will require balancers (reintroduction of the gene product by providing maternal lipids) and the concomitant depletion 
of three or more paralogous enzymes is typically achieved by combining germline mutants with RNAi, thus depleting at least one paralog only incompletely. cgt-1(qa1809); cgt-2(tm1192); cgt-3(RNAi) animals, for instance, retain trace amounts of their product $\mathrm{GlcCer}^{27}$. On the other hand, the depletion of several basic lipid biosynthetic enzymes and their products may generate only larval, rather than embryonic, lethality, and the fat-2(wa17) allele, although lacking all normal PUFAs, is viable ${ }^{10}$.

In principle, any branchpoint further downstream in a biosynthetic pathway can be excluded in the same manner, although branchpoint exclusion may become difficult due to the ability of many complex lipids to interconvert. For instance, the possiblity of mutual interconversion of phosphatidylethanolamine and phosphatidylserine precludes using the phosphatidic acid-processing phospholipid-biosynthetic enzymes phosphatidate phosphatase and cytidine diphosphate-diacylglycerol synthase for branch exclusion of specific phospholipids (Fig. 2). Note that most phospholipids could also be excluded by definitively demonstrating that interrupting the first steps of unsaturated LCFA biosynthesis does not generate the phenotype (see above).

\section{Demonstrating pathway contiguity: connecting simple and complex metabolites (Post- screen I confirmation and analysis: Step 33)}

Demonstrating contiguity between upstream and downstream (branch-specific) enzymes will confirm that the initially identified upstream enzymes exert their effects through this branch and are required for the synthesis of its products, while at the same time defining new functions for specific intermediates and delineating the biosynthetic pathway itself. For instance, our polarity screen revealed that mmBCFAs function in polarity, but it also demonstrated that they function as components of $\mathrm{SLs}^{7}$, which is a finding subsequently confirmed by Zhu et al. ${ }^{28}$.

Evidence for pathway contiguity can be accrued genetically and biochemically. Genetic interactions between hypomorphs, for instance, are expected to show enhancement, and the existence of such interactions would demonstrate that the enzymes operate in the same function but could not prove that they interact in the same pathway. Nulls mutants, which are necessary to address this question rigorously, may not be available given the early essential requirement of many biosynthetic steps. If aspects of the investigated function can be temporally or spatially separated, this can be used to demonstrate functional dependency of upstream on downstream components. Our reversible polarity phenotype, for instance, allowed the temporal separation of its induction and reversion: pathway contiguity could be definitively demonstrated by showing that reversion of a phenotype (induced by depletion of an upstream, FA-biosynthetic enzyme) required the branch-specific downstream enzyme (here, an SL-biosynthetic enzyme) ${ }^{7}$. Rescue of the phenotype by downstream lipid compounds is another definitive demonstration of pathway contiguity. However, whereas the supplementation of simple FAs generally suffices to rescue upstream enzyme losses, exogenous complex lipids may not fully replace the biological functions of their endogenous counterparts (constraints for rescue include but are not limited to, uptake, species-specific compound composition and chirality, and spatial-subcellular-and temporal availability). 
Finally, MS or other quantitative approaches can be used to demonstrate a concomitant relative decrease of the identified branch-specific enzyme's lipid products in animals depleted of their upstream FA biosynthetic enzymes. Given the possibility of interconnection and complementation between different lipid compounds, alterations of absolute levels of specific compounds must, however, be interpreted with caution.

\section{In-depth screening of pathway branches with a focus on complex metabolites: identifying the metabolite species (Screen II and Post-screen II confirmation and analysis: Steps 34- 58)}

Once a specific branch for complex lipid biosynthesis has been identified, it is possible to assemble a more comprehensive RNAi library for this specific branch and add: (1) double and triple RNAi conditions to detect redundant enzymes and (2) RNAi sensitive conditions to detect mild effects (the size of libraries remains similar, although the number of targeted reactions is reduced; Table 2 shows a collection of $C$. elegans SL-biosynthetic enzymes with paralogs). Strong RNAi conditions can be achieved either by using RNAi sensitive strains, such as eri-1(mg366), rrf-3(pk1426) or eri-1(mg366); lin-15B(n744), or by strengthening double-stranded RNA production via increasing the IPTG concentration in agar plates to induce the bacterial polymerase (see 'RNAi feeding plates' in Reagent Setup section). Given the abundance of paralogs (Tables 1,2), the simultaneous perturbation of a redundant gene group using combinatorial RNAi is key. We identified the double knockdown of two CGTs as the endpoint biosynthetic enzymes in our study, and thereby GlcCer as the furthest downstream polarity-affecting lipid species ${ }^{7}$. A forward RNAi screen is unable to detect these genes, and a forward genetic screen is also unlikely to do so because of the high number of animals that would need to be screened to identify a double hit.

The endpoint biosynthetic enzyme is validated, and its product is shown to mediate the function of the other identified biosynthetic enzymes through a combination of germline mutant analysis, specific inhibitors, MS analysis, genetic interaction experiments and/or exogenous compound rescue, as described above (pathway contiguity, Fig. 3). Definitive proof is rescue of the phenotype generated by loss of upstream biosynthetic enzymes via the endpoint product. This may not always be possible owing to specific requirements for complex lipids for certain functions (see above, pathway contiguity). For instance, $C$. elegans uses a $\mathrm{C} 17$ branched-chained long-chain base on its SLs, which is not readily available for purchase. If the above suggested experiments leave doubt, such compounds must either be purified from $C$. elegans or be chemically synthesized. Occasionally, bacteria that produce species-compatible lipids have been used for rescue. For example, a C17isosphinganine has been isolated from Spingobacterium spiritivorum to rescue worms thst are deficient in serine palmitoyltransferases ${ }^{28}$, and mmBCFA-deficient worms can be maintained on Stenotrophomonas maltophilia ${ }^{12}$ (these compounds are not present in OP50, the common $C$. elegans bacterial food source). Beyond demonstrating absence (reduction) of the presumed lipid end product, MS analysis of wild-type versus mutant animals depeleted of the endpoint biosynthetic enzyme (and/or of other enzymes whose losses produce the phenotype) will detect species-specific features of pathway products, the potential appearance of novel compounds, and it will contribute to the chemical characterisation of the endproduct. Lipid extraction processes and MS instrument setup conditions differ for 
different lipids- e.g., for SLs, neutral glycerol lipids and glycerolphospholipids ${ }^{29-34}$. The procedures that are outlined here are targeted to the analysis of $C$. elegans SLs, and specifically to complex SL species, such as Cer, GlcCer and sphingomyelin.

Pathway screens for derivatives: identifying the specific compound (Screen III: Steps 5961)

Complex lipids themselves may be precursors for functionally distinct derivatives. For example, GlcCer, the endproduct identified in our polarity screen, provides the backbone for the large family of GSLs. A lipid glycosylation screen performed in conjunction with a prior lipid biosynthetic pathway screen may thus characterize the specific sugar modification of the identified lipid, and thereby the exact chemical composition of the compound. We used this approach, together with MS analysis, to identify the specific polarity affecting GSL in our study ${ }^{7}$. In this particular case, three negative results permitted the exclusion of a requirement for further sugar modifications of the identified lipid endproduct, thereby defining it as GlcCer itself: (1) the failure to identify the phenotype in the subsequent glycosylation RNAi screen; (2) its absence in a presumed null mutant of beta4mannosyltransferase, the first step sugar-biosynthetic enzyme for the arthro-series of GlcCer-derivatives; and (3) the absence of lactosylceramide and consequently the entire GSL lacto-series in $C$. elegans (demonstrated by MS analysis), restricting the $C$. elegans GSL repertoire to the arthro series. To further refine the structural characteristics of such an end product, additional chemical characteristics that may have emerged in the prior biosynthetic pathway screens via the requirement of modifying enzymes for the function under study, can be confirmed by MS analysis. For instance, the requirement of a FA hydroxylase and of specific LCFA biosynthetic enzymes for polarity allowed us to characterize the endpoint polarity-affecting lipid compound as a hydroxylated GlcCer with a C17 branched chain LCB and a LCFA with a length of likely C22 ${ }^{7}$.

\section{MATERIALS}

\section{REAGENTS}

Lipid-related reagents-Fatty acids of different lengths, numbers and positions of double bonds, hydroxyl groups and branches (Sigma, Matreya, Larodan, Nu-Chek Prep Inc.)

Glycerolipids (mono-, di- and tri-glycerols; Sigma, Avanti Polar Lipids, Matreya, Larodan)

Phospholipids with different headgroups, such as phosphocholine, phosphoethanolamine, phosphoserine, phosphoinosotol and phosphoglycerol (Avanti Polar Lipids, Matreya, Larodan)

Sphingolipids with different sphingoid bases and lipid moieties (Sigma, Biomol, Avanti Polar Lipids, Matreya, Larodan)

Lipid biosynthetic enzyme inhibitors: for examples of fatty acid-, glycerolipid- and phospholipid biosynthetic enzyme inhibitors and references, see Table 1, and for inhibitors of sphingolipid metabolic enzymes see Table 2 (Sigma, Biomol, Matreya, Toronto Research Chemicals) 
Chloroform (Sigma, cat. C7559)

Ethanol (Sigma, cat. no. E7023)

DMSO (Sigma, cat. no. D5879)

C. elegans-specific resources and reagents-C. elegans mutant alleles are available through the Caenorhabditis Genetics Center (http://www.cbs.umn.edu/CGC/) and the National Bioresource Project (http://www.shigen.lab.nig.ac.jp/c.elegans/index.jsp)

RNAi sensitive strains: $r r f-3(p k 1426)$, eri-1(mg366), eri-1(mg366); lin-15B(n744) and eri-1(mg366); lin-35(n745), available from the the Caenorhabditis Genetics Center (http:// www.cbs.umn.edu/CGC/)

RNAi feeding libraries; Source Bioscience: Ahringer library (19,762 bacterial clones), http:// www.lifesciences.sourcebioscience.com/clone-products/non-mammalian/c-elegans/celegans-rnai-library/), Vidal ORFeome library (11,804 bacterial clones, http:// www.lifesciences.sourcebioscience.com/clone-products/non-mammalian/c-elegans/celegans-orfeome-version-11/)

OP50 strain of Escherichia coli (Caenorhabditis Genetics Center, http://www.cbs.umn.edu/ $\mathrm{CGC} /$ )

Yeast extract (BD Biosciences, cat. no. 211931)

Peptone (BD Biosciences, cat. no. 211677)

Tryptone (Acros Organics, cat. no. 611845000)

Bacto Agar (BD Biosciences, cat. no. 214040)

$\mathrm{NaCl}$ (Sigma, cat. no. S7653)

MgSO4 (Sigma, cat. no. M2773)

$\mathrm{CaCl} 2$ (Sigma, cat. no. C3881)

KCl (Sigma, cat. no. P9333)

K2HPO4 (Sigma, cat. no. P3786)

KH2PO4 (Sigma, cat. no. P0662)

$\mathrm{NaOH}$ (Sigma, cat. no. S5881)

Na2HPO4 (Sigma, cat. no. S7907)

Ammonium acetate (NH4OAC, Sigma, cat. no. 431311)

Acetonitrile (CAN, Sigma, cat. no. 34976) 
Methanol (CH3OH, Sigma, cat. no. 34860)

Acetic acid (CH3COOH, Sigma, cat. no. A6283)

Cholesterol (Sigma, cat. no. C8667)

Ampicillin (Sigma, cat. no. A0116)

Cabenicillin (Fisher Scientific, cat. no. BP2648)

Tetracyclin (Fisher Scientific, cat. no. BP912)

IPTG (US Biological, cat. no. I8500)

Sodium hypochlorite solution (4-6\% wt/vol)

! Caution this reagent is hazardous and corrosive. Wear protective gloves and a lab coat.

\section{EQUIPMENT}

Lipid-related equipment-LC-MS instrument, for instance an ABI 4000 Q trap mass spectrometer interfaced to a Dionex U3000 liquid chromatograph equipped with an Astec $\mathrm{NH} 24.5$ X $150 \mathrm{~mm}$, 5 um column.

CentriVap benchtop vacuum concentrator (Savant)

Freeze-drier (Millrock Technology)

Analytical balance (Ohaus)

Probe sonicator (Fisher Scientific Model 505 Sonic Dismembrator)

Ultrasonic water bath (Fisher Scientific Bransonic B-5200R-1)

$13 \times 75 \mathrm{~mm}$ glass test tubes

C. elegans-and RNAi-screen-specific equipment-Dissecting microscope (e.g a Nikon SMZ-U) for phenotype analysis, with or without epifluorescence, depending on the features of the phenotype of interest (e.g. suitable for live analysis of fluorescently-labeled animals on their agar plates). Additional microscopes may be needed, such as one providing, for instance, differential interference contrast (Nomarski; for tracking cell lineage), or a confocal microscope (for analyzing subcellular morphology, e.g., a Leica TCS SL laserscanning microscope). For detailed phenotypic analysis, high magnification, i.e., 630X or $1000 \mathrm{X}$, is usually required.

Bench-top centrifuge for pelleting bacteria and worms (e.g., Eppendorf 5810R)

$37^{\circ} \mathrm{C}$ shaking incubator to grow bacteria (New Brunswick)

Multichannel pipettes (20-200 ml) for dispensing media and bacterial suspensions 
Thermowell sealing tape (aluminum; Corning Costar 6570)

Breatheasy sealing membrane (USA Scientific 9123-6100)

Petri Dishes $60 \times 15$ mm (Genesee, cat. no. 32-105)

Petri Dishes $100 \times 15$ mm (Genesee, cat. no. 32-107)

Petri Dishes $150 \times 15$ mm (Genesee, cat. no. 32-106)

6-well plates $(35 \times 18 \mathrm{~mm}$, Thomas Scientific 6902A01)

12-well plates $(22 \times 18 \mathrm{~mm}$, Thomas Scientific 6902A05)

24-well plates $(15.5 \times 18 \mathrm{~mm}$, Thomas Scientific 6902A09)

48-well plates $(9.8 \times 18 \mathrm{~mm}$, Thomas Scientific 6902A13)

96-well plates (for freezing RNAi clones, Fisher Scientific 08-772-2C)

96-deep-well plates (for liquid-culture of RNAi clones, Fisher Scientific 08-772-1B)

OmniTray plates (Nalge Nunc 242811)

96-pin replicator (Nalge Nunc 250520)

Conical tubes (50 ml; Fisher Scientific, cat. no. 14-432-22)

Collection tubes (15 ml; Fisher Scientific, cat. no. 05-527-90)

Reagent reservoirs (Corning Costar, 83-4870)

Roller

\section{REAGENT SETUP}

\section{Lipid-related reagent setup}

Lipid or inhibitor stock solution: Dissolve lipid or lipid mixture or enzyme inhibitors in an appropriate solvent. Solvents and concentration, as well as preparations vary with different compounds. Examples of stock solutions for some lipids and inhibitors are listed in Table 3.

HPLC mobile phases: Mobile Phase A $=(97: 2: 1 \mathrm{CAN}: \mathrm{CH} 3 \mathrm{OH}: \mathrm{CH} 3 \mathrm{COOH})+5 \mathrm{mM}$ NH4OAC; mobile phase $\mathrm{B}=(99: 1 \mathrm{CH} 3 \mathrm{OH}: \mathrm{CH} 3 \mathrm{COOH})+5 \mathrm{mM}$ NH4OAC. Mobile phases should be freshly prepared daily.

\section{C. elegans-and C. elegans RNAi screen-specific reagent setup and solutions}

LB liquid media: Add $5 \mathrm{~g}$ tryptone, $2.5 \mathrm{~g}$ yeast extract and $5 \mathrm{~g} \mathrm{NaCl}$ per 1 liter of water, and autoclave the mixture. Store at $4{ }^{\circ} \mathrm{C}$ for $2-3$ months. Use directly for growing OP50 bacteria, and add $0.6-1 \mathrm{ml}$ of $100 \mathrm{mg} / \mathrm{ml}$ ampicillin for growing RNAi bacteria. 
LB-antibiotics plates: Add $5 \mathrm{~g}$ tryptone, $2.5 \mathrm{~g}$ yeast extract, $5 \mathrm{~g} \mathrm{NaCl}, 7.5 \mathrm{~g}$ agar per 1 liter of water and autoclave the mixture. Allow the medium to cool to $50-60{ }^{\circ} \mathrm{C}$ before adding 1 $\mathrm{ml}$ of $100 \mathrm{mg} / \mathrm{ml}$ ampicillin and $1 \mathrm{ml}$ of $15 \mathrm{mg} / \mathrm{ml}$ tetracyclin stock solutions. Pour the medium into plates and leave them at room temperature until the agar sets. Store the plates at $4{ }^{\circ} \mathrm{C}$ for $2-3$ months. Use for growing RNAi bacteria.

Nematode growth media (NGM) agar plates: For one liter, add $3 \mathrm{~g} \mathrm{NaCl}, 2.5 \mathrm{~g}$ peptone, $17 \mathrm{~g}$ agar and $975 \mathrm{ml} \mathrm{H} 2 \mathrm{O}$, and autoclave the mixture. Allow the medium to cool to 50$60{ }^{\circ} \mathrm{C}$, and then add $1 \mathrm{ml}$ of cholesterol $(5 \mathrm{mg} / \mathrm{ml}$ in ethanol), $0.5 \mathrm{ml}$ of $1 \mathrm{M} \mathrm{CaCl} 2,1 \mathrm{ml}$ of 1 $\mathrm{M} \mathrm{MgSO} 4,25 \mathrm{ml}$ of 1M Potassium phosphate buffer, $\mathrm{pH}$ 6. Pour into plates and leave them at room temperature until the agar sets. Store at $4{ }^{\circ} \mathrm{C}$ for up to 1 month. Use for growing worms.

RNAi feeding plates: For one liter, add $3 \mathrm{~g} \mathrm{NaCl}, 2.5 \mathrm{~g}$ peptone, $17 \mathrm{~g}$ agar and $975 \mathrm{ml} \mathrm{H} 2 \mathrm{O}$ and autoclave the mixture. Allow the medium to cool to $50-60{ }^{\circ} \mathrm{C}$, and then add $1 \mathrm{ml}$ cholesterol ( $5 \mathrm{mg} \mathrm{ml}^{-1}$ in ethanol), $1 \mathrm{ml}$ of $1 \mathrm{M} \mathrm{CaCl} 2,1 \mathrm{ml}$ of $1 \mathrm{M} \mathrm{MgSO} 4,25 \mathrm{ml}$ of $1 \mathrm{M}$ potassium phosphate buffer, $\mathrm{pH} 6,2.4 \mathrm{ml}$ of $200 \mathrm{mg} / \mathrm{ml}$ IPTG (final concentration: $2 \mathrm{mM}$ ), and $0.5 \mathrm{ml}$ of $50 \mathrm{mg} / \mathrm{ml}$ carbenicillin (final concentration: $25 \mathrm{ug} / \mathrm{ml}$ ). Pour into plates and leave them at room temperature until the agar sets. Make plates fresh 1-3 days before using for RNAi knockdown by feeding. Different IPTG concentration will produce milder or stronger RNAi effects and should be empirically determined for the phenotype under study.

M9 buffer: Disolve $3 \mathrm{~g} \mathrm{KH} 2 \mathrm{PO} 4,6 \mathrm{~g} \mathrm{Na} 2 \mathrm{HPO} 4,5 \mathrm{~g} \mathrm{NaCl}$ in 1 liter of water and autoclave the mixture. Add $1 \mathrm{ml}$ of $1 \mathrm{M} \mathrm{MgSO} 4$ after cooling to room temperature. Use the buffer for washing and aliquotting worms. This solution may be stored at room temperature for months.

Bleach solution: Add $25 \mathrm{ml}$ of $10 \mathrm{~N} \mathrm{NaOH}, 15 \mathrm{ml}$ of household sodium hypochlorite solution and $60 \mathrm{ml}$ water to make $100 \mathrm{ml}$. Bleach solutions lose activity over time. Freshly prepare the solution before using it for synchronizing worms.

CaCl2, 1 M: Add $147.01 \mathrm{~g}$ of $\mathrm{CaCl} 2$ to $500 \mathrm{ml}$ of deionized water and stir until $\mathrm{CaCl} 2$ is completely dissolved, and then bring the volume to 1 liter using deionized water, and autoclave the mixture. Allow the solution to cool to room temperature. This solution may be stored indefinitely at room temperature.

MgSO4, 1 M: Add $246.48 \mathrm{~g}$ of MgSO4 to $500 \mathrm{ml}$ of deionized water, and stir it until $\mathrm{MgSO} 4$ is completely dissolved, and then bring the volume to 1 liter using deionized water and autoclave the mixture. Allow the solution to cool to room temperature. This solution may be stored indefinitely at room temperature.

Potassium phosphate buffer, 1 M (pH 6.0): Add $30.1 \mathrm{~g}$ of K2HPO4 and $118.1 \mathrm{~g}$ of $\mathrm{KH} 2 \mathrm{PO} 4$ to $500 \mathrm{ml}$ of deionized water, and stir it until K2HPO4 and $\mathrm{KH} 2 \mathrm{PO} 4$ are completely dissolved, and then bring the volume to 1 liter using deionized water and autoclave the mixture. Allow the solution to cool to room temperature. This solution may be stored indefinitely at room temperature. 
NaOH, $10 \mathrm{~N}$ : Add $20 \mathrm{~g}$ of $\mathrm{NaOH}$ to $50 \mathrm{ml}$ of deionized water, and stir it until $\mathrm{NaOH}$ is completely dissolved. This solution may be stored indefinitely at room temperature and in plastic containers.

Ampicillin stock solution (100 $\mathbf{~ m g} / \mathbf{m l})$ : Add $1 \mathrm{~g}$ of ampicillin to $10 \mathrm{ml}$ of deionized water to make stock solution. Sterilize the stock solution by filtering with a 0.22 um filter and store 1 -ml aliquots in $1.5-\mathrm{ml}$ tubes at $-20{ }^{\circ} \mathrm{C}$ for $6-12$ months.

Carbenicillin stock solution $(\mathbf{5 0} \mathbf{~ m g} / \mathbf{m l})$ : Add $500 \mathrm{mg}$ of carbenicillin to $10 \mathrm{ml}$ of deionized water to make stock solution. Sterilize the stock solution by filtering with a $0.22-$ um filter, and store $1-\mathrm{ml}$ aliquots in 1.5 -ml tubes at $-20{ }^{\circ} \mathrm{C}$ for $6-12$ months.

Tetracyclin stock solution $(\mathbf{1 5} \mathbf{~ m g} / \mathbf{m l})$ : Add $150 \mathrm{mg}$ of tetracyclin to $10 \mathrm{ml}$ of ethanol to make stock solution. Sterilize the stock solution by filtering with a 0.22 -um filter, and store 1 -ml aliquots in $1.5-\mathrm{ml}$ tubes at $-20^{\circ} \mathrm{C}$ for $6-12$ months.

IPTG stock solution $(\mathbf{2 0 0} \mathbf{~ m g} / \mathbf{m l})$ : Add $2 \mathrm{~g}$ of IPTG to $10 \mathrm{ml}$ of deionized water to make stock solution. Sterilize the stock solution by filtering with a 0.22 -um filter and store $1-\mathrm{ml}$ aliquots in $1.5-\mathrm{ml}$ tubes at $-20^{\circ} \mathrm{C}$ for $6-12$ months.

\section{Equipment setup}

HPLC elution protocol: The protocol comprises the following steps: a 2-min column preequilibration in 9:1 A:B (vol/vol); sample injection; 2-min wash with 100\% A; 12-min linear graddient to $100 \% \mathrm{~B}$; and 1-min post-run column equilibration. The gradient profile is tabulated below, with a flow rate of $1 \mathrm{ml} / \mathrm{min}$ and an injection volume of $5 \mathrm{ul}$.

\begin{tabular}{|l|l|l|}
\hline Time (min) & A\% & B\% \\
\hline-2 & 90 & 10 \\
\hline 0 & 100 & 0 \\
\hline 2 & 100 & 0 \\
\hline 14 & 0 & 100 \\
\hline 15 & 0 & 100 \\
\hline
\end{tabular}

\section{PROCEDURE}

PRE-SCREEN CONFIRMATION AND ANALYSIS: Validation of the phenotype in germline mutants

\section{TIMING 1d to 3 weeks}

1. To cross the strain of the initial RNAi screen (a strain typically carrying a marker, e.g. a GFP label - used as example here) into the germline mutant strain under study, mate $\mathrm{N} 2$ male worms into the marker strain (could also generate a male stock of this strain). 
2. Cross the resultant GFP+ male worms into hermaphrodites of the mutant (a/a) to generate $g f p /+; a /+($ if $g f p$ is integrated into the genome) or $a /+; g f p$ (if $g f p$ is transmitted on an extrachromosomal array) F1 progeny and allow to self.

3. Clone the GFP+ F2 progeny to isolate homozygous GFP+ mutants (expected frequency: $1 / 12$ if the GFP reporter is integrated and $1 / 4$ if on an extrachromosomal array).

4. Evaluate whether the homozygous mutants display the same or similar phenotype as the corresponding RNAi animals (see http://www.wormbook.org for standard genetic procedures).

CRITICAL STEP: Depending on the phenotype, high resolution microscopic analysis (e.g., differential interference contrast and/or confocal microscopic analysis) might be needed, in addition to screening using a dissecting microscope.

\section{PRE-SCREEN CONFIRMATION AND ANALYSIS: exclusion of lipid substrate toxicity or lipid-unrelated mechanisms}

\section{- Timing 2-7d}

5. Prepare lipid stock solution (see Reagent Setup). ?TROUBLESHOOTING

6. Mix lipid stock solution or solvent control with either OP50 or gene-sepcific HT115 RNAi bacteria (e.g., 5-40ul + $120 \mathrm{ul}$, respectively, for each of a 6-well plate; see Step 24, RNAi screen setup). The concentration required for rescue needs to be titrated for each compound or compound mixture.

CRITICAL STEP: Some solvents interfere with RNAi and may therefore produce the false impression of phenotype rescue; it is therefore critical that these experiments be run with the appropriate solvent controls (and/or that results be confirmed in germline mutants).

\section{?TROUBLESHOOTING}

7. Seed the mixture of lipid/solvent and bacteria onto the RNAi or nematode growth medium (NGM) plates, and allow the bacteria to grow for 6-8 $\mathrm{h}$.

CRITICAL STEP: Most lipids are not soluble in aqueous medium, thus they are not well distributed when they are directly applied to a bacterial lawn on NGM plates (some extent of precipitation of the lipid compounds might occur). The Watts lab has described an alternative method in which feeding plates containing lipids are made by mixing the lipids into the agar in the presence of tergitol, a nonionic surfactant ${ }^{35}$.

\section{?TROUBLESHOOTING}

8. Seed or pick worms onto the lipid-supplemented NGM or RNAi plates.

\section{?TROUBLESHOOTING}


9. Score the phenotype.

? TROUBLESHOOTING

SCREEN I: BROAD BIOSYNTHETIC PATHWAY RNAi SCREEN: assembly of an RNAi feeding library

\section{- Timing 3d-1week (depending on familiarity with metabolic pathways)}

10. Expanding from the specific biosynthetic enzyme that was identified, assemble the initial library with the aim to target genes/transcripts involved in a wide range of lipid-related processes and with the emphasis on the basic machinery of FA metabolism. Taking advantage of the high structural conservation of biosynthetic enzymes throughout phylogeny, use several or all of the following approaches to assemble the library: Step 10A, assembly of a list of $C$. elegans lipid metabolism-related genes/proteins; Step 10B, assembly of a list of lipid metabolism-related genes/proteins of all species; Step 10C, assembly of a list of C. elegans orthologs of lipid metabolism-related genes/proteins of other species; and Step 10D, assembly of an expanded list of C. elegans orthologs of lipid metabolism-related proteins.

(A) Assembly of a list of C. elegans lipid metabolism-related genes/ proteins

i. Review experimental data on lipid metabolism in C. elegans navigating from wormbase (where parologs are immediately linked; http://www.wormbase.org) to generate a list of lipidrelated genes in $C$. elegans.

(B) Assembly of a list of lipid metabolism-related genes/proteins of all species

i. Analyze annotated pathway databases such as the LIPID MAPS (http://www.lipidmaps.org/pathways/index.html) and the KEGG pathways (http://www.genome.jp/kegg/ pathway.html), which provide systematically aligned metabolic pathways, for a list of lipid-related molecules of all species (see en.wikipedia.org/wiki/

List_of_biological_databases and http://www.nature.com/ scitable/content/genomics-databases-744357 for comprehensive lists of biological databases).

(C) Assembly of of a list of C. elegans orthologs of lipid metabolismrelated proteins of other species.

i. Search databases such as AceView (http://ncbi.nlm.gov/ieb/ research/assembly/) and iHOP (http://www.ihop-net.org/ UniPub/iHop/) with links to multiple organisms, and speciesspecific databases, such as the Saccharomyces Genome Database (http://www.yeastgenome.org), Flybase (http:// www.flybase.org), the Mouse Genome Database (http:// 
www.informatics.jax.org) and the Human Genome Database (http://www.gdb.org), to identify C. elegans orthologs of nonC.elegans proteins.

(D) Assembly of an expanded list of C. elegans orthologs of lipid metabolism-related proteins.

i. Perform protein BLAST searches against the C. elegans databases to identify $C$. elegans orthologs of non-C.elegans proteins not traced with the above search engines, and perform searches inside the $C$. elegans database to identify more distantly related family members.

11. Pick RNAi clones of these candidate genes from the genome-wide RNAi feeding libraries onto LB plates containing the appropriate selective antibiotics, and grown overnight at $37^{\circ} \mathrm{C}$.

12. Inoculate bacteria into $120 \mathrm{ul}$ of LB media plus $100 \mathrm{mg} / \mathrm{ml}$ ampicillin in a 96well plate, allow the bacteria to grow overnight in a shaking incubator at 220 r.p.m. at $37{ }^{\circ} \mathrm{C}$, and then add $30 \mathrm{ul}$ of $80 \%$ glycerol and keep it in a $-80{ }^{\circ} \mathrm{C}$ freezer to generate a frozen stock of the library.

\section{SCREEN I: BROAD BIOSYNTHETIC PATHWAY RNAi SCREEN: worm synchronization \\ TIMING 3-6 d}

13. Place 10-15 young adult hermaphrodites on each of ten 100-mm standard NGM plates seeded with OP50, and allow them to grow at $20{ }^{\circ} \mathrm{C}$ until the plate is full of gravid adults, but the worms are not starved (i.e., some of the OP50 lawn remains on the plate).

14. Wash the worms from the plates using $\mathrm{M} 9$ ( $1-3 \mathrm{ml}$ per plate), and collect the M9 worm suspension into a $15-\mathrm{ml}$ conical tube.

15. Centrifuge the collected worms at 1,500 r.p.m. for $5 \mathrm{~min}$ at room temperature and carefully decant the supernatant.

16. Add $3.5 \mathrm{ml}$ of cold $\mathrm{M} 9$ and $1.5 \mathrm{ml}$ of sodium hypochlorite solution to the worm pellet, mix by inversion and vortex the mixture.

17. Swirl the solution every minute or so until almost all adults have dissolved (it takes 6-8 min depending on the number of worms in each tube). Immediately fill the tube with M9 and centrifuge at 1,500 r.p.m. at room temperature for 2 min. Carefully decant the bleach solution without disturbing the worm embryo pellet.

18. Wash the embryo pellet using 5-10 $\mathrm{ml}$ of $\mathrm{M} 9$ buffer three times to remove bleach.

19. Resuspend the washed embryos in 5-10 $\mathrm{ml}$ of $\mathrm{M} 9$.

20. Allow the collected embryos to hatch in $\mathrm{M} 9$ by rotating them at $20^{\circ} \mathrm{C}$ for $12-20$ h. 
CRITICAL STEP Note that both the eggs from step 19 and L1 larvae from step 20 can be used directly for an L1-initiated RNAi feeding screen.

21. Plate the synchronized larvae in M9 onto NGM plates seeded with OP50 (depending on the amount of larvae, they can be plated onto several NGM plates to ensure a well-fed condition).

22. Incubate the plates at $20^{\circ} \mathrm{C}$ for $36-48$ hours.

23. Wash the worms off the plates using M9 buffer three times before pipetting onto RNAi feeding plates for screening (for plate preparation, see step 26).

\section{SCREEN I: BROAD BIOSYNTHETIC PATHWAY RNAi SCREEN: RNAi feeding and phenotype scoring}

\section{- TIMING 3-6 d}

24. The day before setting up RNAi feeding experiments, inoculate bacteria from step 12 into 500-800 ul (50-200 ul final volume is required for each individual feeding experiment, depending on the type of plate used: 6-, 24- or 96 well) of LB media plus $100 \mathrm{mg} / \mathrm{ml}$ ampicillin in a 96-deep well plate, and allow them to grow overnight in a shaking incubator at 220 r.p.m. at $37^{\circ} \mathrm{C}$.

CRITICAL STEP: It is important to use freshly grown bacteria to achieve the full RNAi effect.

25. The following morning, induce transcription of double-stranded RNA by adding 50-200 ul of the bacterial cultures from Step 24 to each RNAi plate per well (containing IPTG, see 'RNAi feeding plates' in Reagent Setup section). For knock downs of multiple genes, add an equal volume of each bacterial feeding clone to the plates.

CRITICAL STEP: RNAi has an intrinsic variability, therefore, it is essential to set up replicates for every clone. In each experimental run, at least one positive and one negative control are included (Step 27).

26. After 10-12 h, for parentally induced RNAi (where the F1 generation is scored), pipette L3-L4 worms from Setp 23 onto each RNAi well per plate (typically 510 worms in a volume of $10 \mathrm{ul}$ of M9 buffer for six-well plates); for RNAi induced in the same generation, pipette eggs or L1s from Step 20 (typically 50100 worms in a volume of 10-20 ul of M9 buffer for six-well plates).

CRITICAL STEP: Experiments may need to be repeated with larger number of worms if the brood size is low.

27. Scoring depends on the screen set-up and the nature of the phenotype of interest. To score morphological F1 phenotypes scored on a dissecting microscope, day 2 is optimal for scoring embryonic phenotypes, and day 3 is optimal for scoring larval phenotypes. If maternal effects are being scored, also inspect the F3 generation. Suitable bacterial control RNAi clones should be included for each set of RNAi experiments. The optimal positive control is a clone that copies the phenotype of interest, and the optimal negative control is a clone that produces 
an unrelated phenotype or no dectatable phenotype (e.g., targets a non $C$. elegans gene).

CRITICAL STEP: Steps 13-27 constitute one cycle of RNAi screening. Depending on numbers and time required for scoring, several overlapping cycles of screening can be carried out per week.

\section{? Troubleshooting}

\section{CONFIRMATION AND ANALYSIS OF RESULTS OF SCREEN I: demonstration of pathway linearity $\bigcirc$ TIMING 3-7 d}

28. Identify the enzyme catalyzing the reaction immediately upstream or downstream of the enzyme identified by RNAi phenocopy in the above screens (use resources indicated in Step 10).

29. Perform RNAi with this enzyme as described in Steps 13-27, to demonstrate copy of the phenotype.

\section{?TROUBLESHOOTING}

\section{CONFIRMATION AND ANALYSIS OF RESULTS OF SCREEN I: Validation of branch selection - TIMING 3 to 4weeks (depending on options chosen)}

30. Pobe the validity of a branch-specific positive result by either one or several of the following steps: Step 30A, confirmation of phenotype in germline mutants; Step 30B, demonstration of phenocopy by small molecule inhibitors; and Step 30C, rescue of phenotype via downstream lipid product(s).

(A) Confirmation of phenotype in germline mutants

i. Use procedures described in Steps 1-4 to generate and analyze the branch-specific germline mutant.

(B) Demonstration of phenocopy by small-molecule inhibitors

i. Dilute the inhibitor stock solution or solvent control with OP50 bacteria to the desired volume of, for instance, 150 ul per well of a six-well plate (typically a $4-25$ time dilution). The optimum concentration to produce the phenotype of interest needs to be titrated for each compound.

ii. Seed the mixture of solution onto the NGM plates and let OP50 bacteria grow for 6-8 hours (see 'NGM agar plates' in Reagent Setup section)

CRITICAL STEP: Some precipitation may occur owing to water insolubility (see Steps 5-9, analysis of lipid compounds, for caveats with regards to lipophilic substances).

iii. Seed eggs, larvae or adults of the strain used to carry out the initial screen onto the plates.

iv. Score the phenotype. 


\section{? Troubleshooting}

(C) Rescue of phenotype via downstream lipid product(s).

i. Use procedures described in Steps 5-9 to rescue the branchspecific enzyme defect with downstream lipids.

CRITICAL STEP: This approach may not be an option, as only complex lipids (rather than FAs) will rescue branch-specific enzyme knockdowns-which may require species-specific compounds that are not readily identified or available (see Experimental design). Failure of rescue with complex lipids must thus be interpreted with caution.

\section{CONFIRMATION AND ANALYSIS OF RESULTS OF SCREEN I: exclusion of lipid classes or branches TIMING 1-4weeks (depending on availability of mutants and their characterization); this is optional if a branch-specific enzyme was identified, but required in the absence of such a positive result)}

31. Generate severe double or triple loss-of-function conditions for paralogous enzymes (ideally null mutants) to abolish enzymatic reactions for the purpose of pathway branch exclusions, using a combination of standard genetic procedures, RNAi and specific enzyme inhibitors (see Steps 1-4 for an example of a simple cross; Steps 13-27 for RNAi; Step 30B, for treatment with inhibitors; and http:// www.wormbook.org for standard genetic procedures, including the introduction of genetic balancers into lethal mutants). Some double and triple mutants are already available at http://www.cbs.umn.edu/CGC/ or they may have been generated for specific studies (e.g., fat-6(tm331); fat-7(wa36). fat-7(wa36) fat-5(tm420). fat-6(tm331); fat-5(tm420). acl-4(xh10) acl-5(xh9). cgt-3(tm504); +/DnT1; cgt-1(qa1809)/DnT1; cgt-2(tm1097). cgt-3(tm504); +/DnT1; cgt-1(qa1809)/DnT1; cgt-2(tm1192) double and triple mutants ${ }^{13}$ 26, 27 ; see Fig. $1)$.

32. Examine the mutant for copies of the phenotype

CRITICAL STEP: To definitively exclude a class or branch, MS analysis is additionally required to demonstrate absence of the product of the enzymatic reaction in question: see Steps 37-58 below for MS analysis (such data may, however, be already available for some of these mutants).

\section{CONFIRMATION AND ANALYSIS OF RESULTS OF SCREEN I: Demonstration of pathway contiguity $\bigcirc$ TIMING 2-4 weeks}

33. Show that the function of upstream biosynthetic enzymes is mediated by downstream biosynthetic enzymes/compounds by one or several of the following approaches: Step 33A, genetic interactions; Step 33B, phenotype rescue via downstream branch product(s); Step 33C, biochemical analysis of lipids (MS).

(A) Genetic interactions 
i. Create double loss-of-function mutants, mutant/RNAi-, double-RNAi- or mutant/inhibitor/RNAi animals whose alterations concomitantly deplete one FA and one complex lipid biosynthetic enzyme (see Steps 1-4 for an example of a simple cross; Steps 13-27 for RNAi; Step 30B, for treatment with inhibitors). To score for the expected enhancement, aim for mild loss-of-function conditions. Titrate mild RNAi conditions by reducing IPTG plate concentration and/or by diluting RNAi bacteria with parent bacteria until a penetrance of $0-50 \%$ is achieved.

ii. Score the phenotype.

CRITICAL STEP: Consider on a case-by-case basis whether it is worth investing time into genetic interaction experiments, as haploinsufficiency, as well as dosage-dependent nonallelic noncomplementation, is thought to occur only rarely in $C$. elegans $^{36}$. We did, however, find them helpful when examining metabolic enzymes and dosage-dependent processes, and we have also used heterozygous germline mutants for this purpose ${ }^{7}$.

\section{? Troubleshooting}

(B) Phenotype rescue via downstream branch product(s)

i. Refer to Step 30C (including caveats) and use procedures described in Steps 5-9 to rescue an upstream biosynthetic enzyme defect with a downstream branch-specific lipid.

(C) Biochemical analysis of lipids by MS

i. Demonstrate similar lipid profiles with regard to the missing lipid compound in RNAi animals/mutants with defects in upstream versus downstream biosynthetic pathway enzymes (see Steps 37-58 below).

\section{SCREEN II: COMPREHENSIVE PATHWAY BRANCH RNAi SCREEN: assembly of an RNAi feeding library $\bigcirc$ Timing $3-7 d$}

34. Construct this smaller library (compared to the broad library assembled for screen I) as comprehensively as possible and include the simultaneous depletion of paralogs to effectively interfere with at least all those enzymatic reactions that could generate an endproduct (rather than an intermediate) and thus the searched-for lipid compound (as described in Steps 10-12).

\section{SCREEN II: COMPREHENSIVE PATHWAY BRANCH RNAi SCREEN: RNAi screen procedure - Timing 6-12d}

35. Synchronize worms, set up RNAi feeding experiments and score phenotypes, as described for screen I (Steps 13-27). 
CRITICAL STEP: The addition of double and triple RNAi for the depletion of redundant enzymes and/or of strong RNAi conditions to optimize enzyme depletion is critical for this round of screening (see Experimental Design).

\section{CONFIRMATION AND ANALYSIS OF RESULTS OF SCREEN II: Validation of the identified furthest downstream lipid biosynthetic enzyme and its product $\bigcirc$ Timing 2-4 weeks}

36. Validate the presumed endpoint lipid biosynthetic enzyme and its lipid compound and demonstrate that they constitute the endpoint biosynthetic enzyme/compound pair by several of the following procedures: Step 36A, scoring for phenocopy in the corresponding germline mutant; Step 36B, scoring for phenocopy by the specific inhibitor of the identified enzyme; Step 36C, screening for genetic interaction of the identified enzyme with any or all other enzymes of the pathway; Step 36D, rescue of the loss-of-function phenotype(s) of the endpoint biosynthetic enzyme and/or of any upstream enzymes by the presumed lipid end product; and Step 36E, MS analysis of the loss-of-function mutant/RNAi animal(s) that display the phenotype to demonstrate absence (reduction) of the presumed lipid end product.

(A) Scoring for phenocopy in the corresponding germline mutant

i. $\quad$ Use procedures described in Steps 1-4.

(B) Scoring for phenocopy by the specific inhibitor of the identified enzyme

i. Use procedures described in Step 30B.

(C) Screening for genetic interaction of the identified enzyme with any or all other enzymes of the pathway.

i. Use procedures described in Step 33A.

(D) Rescue of the loss-of-function phenotype(s) of the endpoint biosynthetic enzyme and/or of any upstream enzymes by the presumed lipid end product.

i. Refer to Step 30C and use procedures described in Steps 5-9.

(E) MS analysis of the loss-of-function mutant/RNAi animal(s) that display the phenotype to demonstrate the absence (reduction) of the presumed lipid end product.

i. $\quad$ See Steps 37-58.

\section{CONFIRMATION AND ANALYSIS OF RESULTS OF SCREEN II: Lipid profile analysis Timing 1-3 weeks}

37. Preparation of wild-type and mutant/RNAi worms depleted of the endpoint biosynthetic enzyme for lipid extraction. Set up 150mm RNAi (for RNAi depletion) or NGM culture plates (for germline mutants) to grow a sufficiently large amount of worms for lipid extraction, aiming at a final worm pellet of at 
least 200 ul. If L1 larvae are to be analyzed (a likely scenario given the frequent L1 lethality of lipid biosynthetic enzyme mutants/RNAi animals), $600-800$ such plates may be required. As controls, set up equal amounts of synchronized wild-type L1 larvae, which are grown on the same bacterial strain (E. coli HT115).

38. Collect worms by rinsing the RNAi plates with M9 buffer and letting animals sediment at $4^{\circ} \mathrm{C}$.

39. Subject worm pellets to three cycles of freezing in liquid nitrogen for 30-60 minutes and thawing on ice for $30 \mathrm{~min}$, followed by sonication (10 s each time, 3-5 times for each cycle).

40. Combine samples with the same genotype into one tube, lyophilize the worm suspension using a freeze-drier and measure dry weight using an analytical balance.

41. Add methanol, chloroform and water in a 2:1:1 ratio (e.g. $2 / 1 / 1 \mathrm{ml}$ ) to each sample ( 40-60 mg dried worm pellet).

42. Spike $100 \mu \mathrm{l}$ of internal standard mixture (e. g., a SL standard mixture).

43. Mix by vortexing for 5 minutes to achieve a single-phase cloudy solution.

44. Incubate the samples at $48^{\circ} \mathrm{C}$ overnight in a heating block.

45. Add $1.0 \mathrm{ml}$ of $1 \mathrm{M} \mathrm{KOH}$ into each sample after cooling to room temperature.

46. Sonicate the samples in a bath sonicator for $5 \mathrm{~min}$.

47. Incubate at $37^{\circ} \mathrm{C}$ for $2 \mathrm{~h}$.

48. Remove a 1-ml aliquot of the extract and dry it under vacuum for long-chain base analysis when necessary.

49. Add $600 \mu \mathrm{l}$ of glacial acetic acid to neutralize the remaining solution.

50. Add $4 \mathrm{ml}$ of water and $2 \mathrm{ml}$ of chloroform, and shake for $2 \mathrm{~min}$.

51. Centrifuge at $1,000 \mathrm{~g}$ for $10 \mathrm{~min}$ at $20^{\circ} \mathrm{C}$ and discard the upper layer.

52. Collect the lower layer for analysis, and reduce the volume to $\sim 100$ ul using a CentriVap Benchtop Vacuum Concentrator.

PAUSE POINT The samples can be stored at $-20^{\circ} \mathrm{C}$ for $2-3$ months.

53. Reconstitute the samples to a final volume of $1.0 \mathrm{ml}$ with a 9:1 mixture of HPLC mobile phase A and B (see Reagent Setup section).

CRITICAL STEP: Glassware rather than plastic should be used for all extraction procedure steps that use organic solvents, in order to avoid extracting molecules from the plastic that could interfere with the biochemical analysis of lipids.

54. L C-MS. Carry out HPLC using Dionex U3000 liquid chromatograph equipped with an Astec NH2 column (4.5 mm $\times 150 \mathrm{~mm}, 5-\mu \mathrm{m}$ particles); mobile phases 
A and B are prepared as described in Reagent Setup section, and elution protocol is described in Equipment Setup section.

55. Set up the following conditions for MS (ABI 4000 Qtrap): Ion Spray voltage: $4500 \mathrm{~V}$; ion spray temperature: $400^{\circ} \mathrm{C}$; gas pressure: 5-20 p.s.i; program to monitor the $[\mathrm{M}+\mathrm{H}] \rightarrow 250.2 \mathrm{u}$ ion of the d17:1 C. elegans specific sphingoid base; ceramide/hydroxyceramide, hydroxyglucoceramide, and sphingomyelin/ hydroxysphingomyelin compounds were monitored in three periods according to normal phase elution time of the internal standards.

56. Inject $5 \mu \mathrm{l}$ of each $1 \mathrm{ml}$ extract from Step 53 .

57. Calculate concentrations for each compound from the response ratio of compound area/internal standard area of the corresponding period per dry weight of the sample.

58. Compare the lipid composition of mutant/RNAi animals with control animals.

CRITICAL STEP: It is not always useful to generate whole lipidomic profiles, as this may decrease the sensitivity for a lipid species of interest.

\section{SCREEN III: METABOLIC PATHWAY RNAi SCREEN FOR DERIVATIVES (optional): Assembly of an RNAi feeding library $\bigcirc$ Timing 3-7d}

59. Construct an RNAi library from enzymes known or expected to biochemically modify the identified lipid end product-for example, enzymes functioning in lipid glycosylation (for library assembly see Steps 10-12). As described in Step 34 (and library size permitting) aim for concomittant depeletion of paralogs and a broad range of depletion conditions.

\section{SCREEN III: METABOLIC PATHWAY RNAi SCREEN FOR DERIVATIVES (optional): RNAi screen procedure 0 Timing $6-12 \mathrm{~d}$}

60. Synchronize worms, set up RNAi feeding experiments and score phenotypes, as described for screen I (Steps 13-27).

\section{CONFIRMATION AND ANALYSIS OF RESULTS OF SCREEN III: validation of the endpoint lipid-modifying enzyme and chemical characterization of the endpoint lipid 0 Timing 2-4 weeks}

61. Proceed according to the corresponding Steps outlined for screen II (36-58).

\section{?TROUBLESHOOTING}

Trouble shooting advice can be found in Table 4

TIMING

PRE-SCREEN CONFIRMATION AND ANALYSIS:

Steps 1-4, phenotype validation in germline mutants: $1 \mathrm{~d}$ to 3 weeks

Steps 5-9, exclusion of lipid substrate toxicity or lipid-unrelated mechanisms: $2-7 \mathrm{~d}$ 


\section{SCREEN I: BROAD BIOSYNTHETIC PATHWAY RNAi SCREEN:}

Steps 10-12, assembly of an RNAi feeding library: 3d to1week

Steps 13-23, worm synchronization: 3-6 d

Steps 24-27, RNAi feeding setup and scoring the phenotype: 3-6 d

CONFIRMATION AND ANALYSIS OF RESULTS OF SCREEN I (optional)

Steps 28 and 29, demonstration of pathway linearity: 3-7 d

Step 30, validation of branch selection: $3 \mathrm{~d}$ to 4 weeks

Steps 31 and 32, exclusion of lipid classes or branches: 1-4weeks

Step 33, demonstration of pathway contiguity: 2-4 weeks

SCREEN II: COMPREHENSIVE PATHWAY BRANCH RNAi SCREEN

Step 34, assembly of an RNAi feeding library: 3-7d

Step 35, RNAi screen procedure: 6-12d

\section{CONFIRMATION AND ANALYSIS OF RESULTS OF SCREEN II}

Step 36, validation of downstream lipid-biosynthetic enzyme and its product: 2-4 weeks

Steps 37-58, lipid profile analysis: 1-3 weeks

SCREEN III: METABOLIC PATHWAY RNAi SCREEN FOR DERIVATIVES (optional)

Step 59, assembly of an RNAi feeding library: 3-7d

Step 60, RNAi screen procedure: 6-12d

\section{CONFIRMATION AND ANALYSIS OF RESULTS OF SCREEN III}

Step 61, validation of the endpoint lipid-modifying enzyme and chemical characterization of the endpoint lipid: 2-4 weeks

\section{ANTICIPATED RESULTS}

In this era of fully sequenced genomes, biosynthetic enzyme genes are frequently identified in genome-wide screens (e.g., RNAi screens) that investigate specific cell biological functions. This information is often excluded from further analysis, because such 'housekeeping genes' typically contribute to the synthesis of a vast array of different compounds. Systematic follow-on pathway screens, as described here, are expected to identify the one specific metabolite that mediates their contribution to the investigated function, and to additionally map out the entire pathway leading to its synthesis. Such screens will therefore also identify multiple intermediate enzymes and their respective products as required for the function under study. With sufficient screen depth and the addition of screens for derivatives, biosynthetic pathway screens, together with MS-based biochemical analyses, are able to identify not only the compound species whose loss causes the functional defect, but also its exact chemical characteristics. Biosynthetic pathway screens can also be devised upfront as 'metabolic RNAi screens' to investigate the 
contribution of nonproteinaceious compounds to a specific biological function. The prime purpose of these screens is the discovery of novel, non-metabolic functions of nontemplatederived metabolites such as lipids (themselves no targets for knockouts or knockdowns), although they also extend the already well-established knowledge about their metabolic functions. It is hoped that such screens will harness the power of unbiased genetic/RNAi screens to open the field of metabolomics for the in vivo characterization of the largely unknown non-metabolic roles of biosynthetic intermediates.

\section{Supplementary Material}

Refer to Web version on PubMed Central for supplementary material.

\section{Acknowledgments}

C. elegans strains were provided by G. Ruvkun (Massachusetts General Hospital, Harvard Medical School, Boston, Massachusetts, USA), S. Mitani (National Bioresource Project Japan) and the Caenorhabditis Genetics Center (NIH Center for Research Resources). We thank J. Moore (Avanti Polar Lipids) and Edward Membreno for contributions to LC/MS and C. elegans maintenance, respectively. We thank H. Weinstein and R. Kleinman for ongoing support. This work was supported by US National Institute of Health grant GM078653, Massachusetts General Hospital IS Funding and a Mattina R. Proctor Award to V.G.

\section{References}

1. van Meer G, Voelker DR, Feigenson GW. Membrane lipids: where they are and how they behave. Nat. Rev. Mol. Cell Biol. 2008; 9:112-124. [PubMed: 18216768]

2. Blom T, Somerharju P, Ikonen E. Synthesis and biosynthetic trafficking of membrane lipids. Cold Spring Harb Perspect Biol. 2011; 3:a004713. [PubMed: 21482741]

3. Wenk MR. Lipidomics: new tools and applications. Cell. 2010; 143:888-895. [PubMed: 21145456]

4. Ivanova PT, Milne SB, Myers DS, Brown HA. Lipidomics: a mass spectrometry based systems level analysis of cellular lipids. Curr. Opin. Chem. Biol. 2009; 13:526-531. [PubMed: 19744877]

5. Halabalaki M, Vougogiannopoulou K, Mikros E, Skaltsounis AL. Recent advances and new strategies in the NMR-based identification of natural products. Curr. Opin. Biotechnol. 2014; 25C: $1-7$.

6. Jungmann JH, Heeren RM. Emerging technologies in mass spectrometry imaging. J. Proteomics. 2012; 75:5077-5092. [PubMed: 22469858]

7. Zhang $\mathrm{H}$, et al. Apicobasal domain identities of expanding tubular membranes depend on glycosphingolipid biosynthesis. Nat. Cell. Biol. 2011; 13:1189-1201. [PubMed: 21926990]

8. Breslow DK, Weissman JS. Membranes in balance: mechanisms of sphingolipid homeostasis. Mol. Cell. 2010; 40:267-279. [PubMed: 20965421]

9. Hannun YA, Obeid LM. Many ceramides. J. Biol. Chem. 2011; 286:27855-27862. [PubMed: 21693702]

10. Watts JL, Browse J. Genetic dissection of polyunsaturated fatty acid synthesis in Caenorhabditis elegans. Proc. Natl. Acad. Sci. U S A. 2002; 99:5854-5859. [PubMed: 11972048]

11. Ashrafi K, et al. Genome-wide RNAi analysis of Caenorhabditis elegans fat regulatory genes. Nature. 2003; 421:268-272. [PubMed: 12529643]

12. Kniazeva M, Crawford QT, Seiber M, Wang CY, Han M. Monomethyl branched-chain fatty acids play an essential role in Caenorhabditis elegans development. PLoS Biol. 2004; 2:E257. [PubMed: 15340492]

13. Brock TJ, Browse J, Watts JL. Genetic regulation of unsaturated fatty acid composition in C elegans. PLoS Genet. 2006; 2:e108. [PubMed: 16839188]

14. Entchev EV, et al. LET-767 is required for the production of branched chain and long chain fatty acids in Caenorhabditis elegans. J. Biol. Chem. 2008; 283:17550-17560. [PubMed: 18390550] 
15. Elle IC, Olsen LC, Pultz D, Rodkaer SV, Faergeman NJ. Something worth dyeing for: molecular tools for the dissection of lipid metabolism in Caenorhabditis elegans. FEBS Lett. 2010; 584:2183-2193. [PubMed: 20371247]

16. Barros AG, Liu J, Lemieux GA, Mullaney BC, Ashrafi K. Analyses of C. elegans fat metabolic pathways. Methods Cell Biol. 2012; 107:383-407. [PubMed: 22226531]

17. Papan C, et al. Systematic screening for novel lipids by shotgun lipidomics. Anal. Chem. 2014; 86:2703-2710. [PubMed: 24471557]

18. Vance, DE., Vance, JE. Biochemistry of lipids, lipoproteins and membranes. 5. Elsevier; 2008.

19. Xu YH, Barnes S, Sun Y, Grabowski GA. Multi-system disorders of glycosphingolipid and ganglioside metabolism. J. Lipid Res. 2010; 51:1643-1675. [PubMed: 20211931]

20. Kniazeva M, Euler T, Han M. A branched-chain fatty acid is involved in post-embryonic growth control in parallel to the insulin receptor pathway and its biosynthesis is feedback-regulated in C. elegans. Genes Dev. 2008; 22:2102-2110. [PubMed: 18676815]

21. Shi Y, Burn P. Lipid metabolic enzymes: emerging drug targets for the treatment of obesity. Nat. Rev. Drug Discov. 2004; 3:695-710. [PubMed: 15286736]

22. Hess D, Chisholm JW, Igal RA. Inhibition of stearoylCoA desaturase activity blocks cell cycle progression and induces programmed cell death in lung cancer cells. PLoS One. 2010; 5:e11394. [PubMed: 20613975]

23. Kamphorst JJ, et al. Hypoxic and Ras-transformed cells support growth by scavenging unsaturated fatty acids from lysophospholipids. Proc. Natl. Acad. Sci. U S A. 2013; 110:8882-8887. [PubMed: 23671091]

24. Delgado A, Casas J, Llebaria A, Abad JL, Fabrias G. Inhibitors of sphingolipid metabolism enzymes. Biochim. Biophys. Acta. 2006; 1758:1957-1977. [PubMed: 17049336]

25. Albi E, Viola Magni M. Sphingolipid Metabolism Inhibitors and Cell Function. The Open Enzyme Inhib. J. 2008; 1:72-79.

26. Ohba Y, et al. Mitochondria-type GPAT is required for mitochondrial fusion. EMBO J. 2013; 32:1265-1279. [PubMed: 23572076]

27. Marza E, Simonsen KT, Faergeman NJ, Lesa GM. Expression of ceramide glucosyltransferases, which are essential for glycosphingolipid synthesis, is only required in a small subset of C. elegans cells. J. Cell. Sci. 2009; 122:822-833. [PubMed: 19240113]

28. Zhu H, Shen H, Sewell AK, Kniazeva M, Han M. A novel sphingolipid-TORC1 pathway critically promotes postembryonic development in Caenorhabditis elegans. Elife. 2013; 2:e00429. [PubMed: 23705068]

29. Krank J, Murphy RC, Barkley RM, Duchoslav E, McAnoy A. Qualitative analysis and quantitative assessment of changes in neutral glycerol lipid molecular species within cells. Methods Enzymol. 2007; 432:1-20. [PubMed: 17954211]

30. Ivanova PT, Milne SB, Byrne MO, Xiang Y, Brown HA. Glycerophospholipid identification and quantitation by electrospray ionization mass spectrometry. Methods Enzymol. 2007; 432:21-57. [PubMed: 17954212]

31. Deems R, Buczynski MW, Bowers-Gentry R, Harkewicz R, Dennis EA. Detection and quantitation of eicosanoids via high performance liquid chromatography-electrospray ionization-mass spectrometry. Methods Enzymol. 2007; 432:59-82. [PubMed: 17954213]

32. Sullards MC, et al. Structure-specific, quantitative methods for analysis of sphingolipids by liquid chromatography-tandem mass spectrometry: "inside-out" sphingolipidomics. Methods Enzymol. 2007; 432:83-115. [PubMed: 17954214]

33. Garrett TA, Guan Z, Raetz CR. Analysis of ubiquinones, dolichols, and dolichol diphosphateoligosaccharides by liquid chromatography-electrospray ionization-mass spectrometry. Methods Enzymol. 2007; 432:117-143. [PubMed: 17954215]

34. McDonald JG, Thompson BM, McCrum EC, Russell DW. Extraction and analysis of sterols in biological matrices by high performance liquid chromatography electrospray ionization mass spectrometry. Methods Enzymol. 2007; 432:145-170. [PubMed: 17954216]

35. Deline ML, Vrablik TL, Watts JL. Dietary supplementation of polyunsaturated fatty acids in Caenorhabditis elegans. J. Vis. Exp. 2013 
36. Yook, K. Complementation. WormBook, ed. The C. elegans Research Community. 2005. http:// dx.doi.org/10.1895/wormbook.1.24.1 


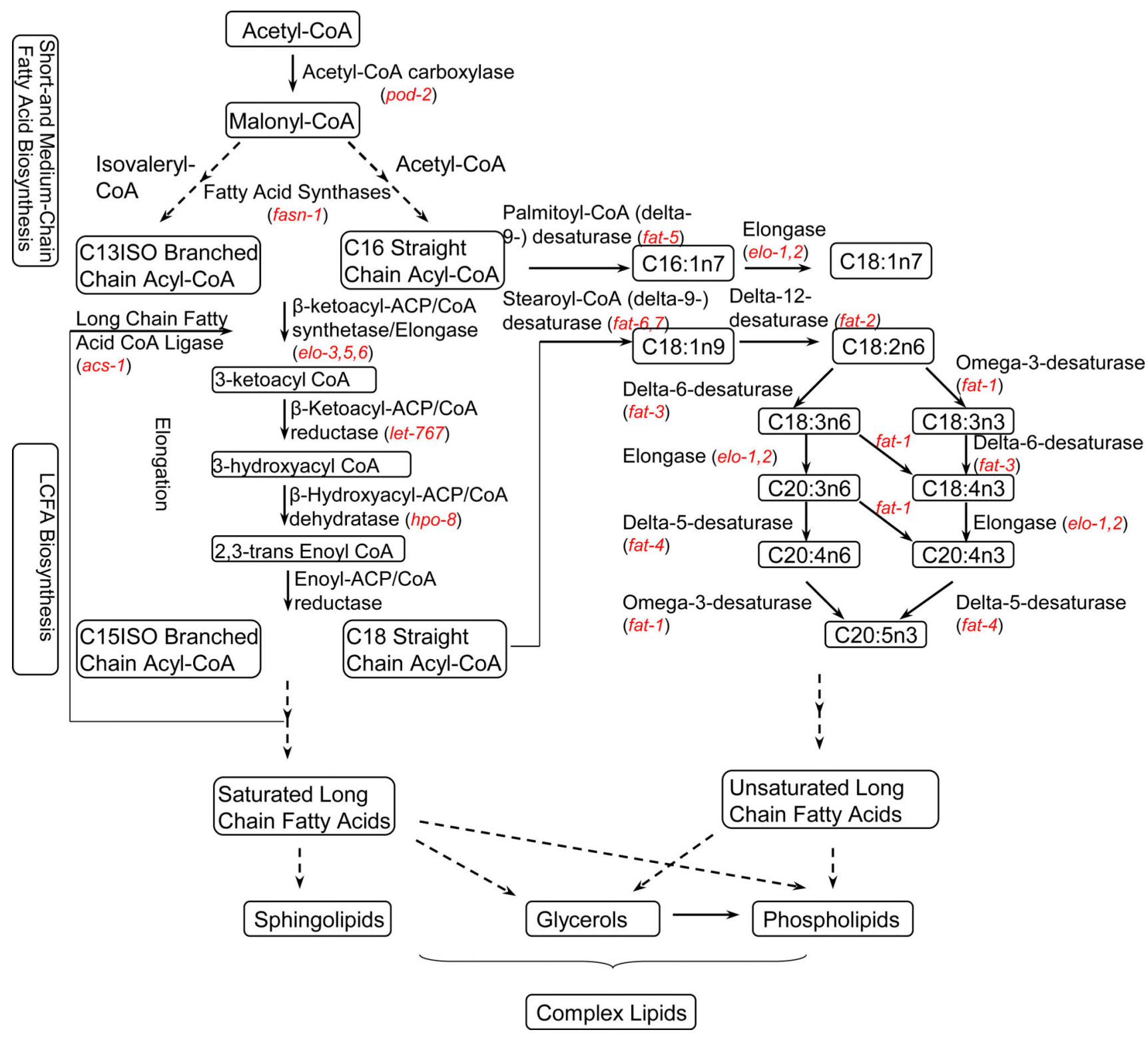

Figure 1. Conserved fatty-acid biosynthetic pathways. Top: Short- and medium-chain-fatty-acid (FA) biosynthesis

The biosynthesis of fatty acids FAs begins with a primer molecule, usually acetic acid, in the form of its CoA ester or attached to an acyl carrier protein (ACP), which forms malonylCoA by the activity of the enzyme acetyl-CoA carboxylase. Successive chain elongation, executed by adding malonyl-CoA as a chain extender to the single primer molecules through repeated multiple-step cycles catalyzed by the multifunctional FA synthase builds saturated short-chain FAs or medium-chain FAs. Acetyl-CoA is shown as primer example for evennumbered straight-chain FAs, and isovaleryl-CoA for odd-numbered monomethyl branchedchain FAs. Middle left: Long-chain-fatty-acid (LCFA) biosynthesis. Straight and branched medium-chain FAs are further elongated in two-carbon units using malonyl-CoA as donor through repeated four-step (condensation, reduction, dehydration and reduction) cycles catalyzed by an enzyme complex with four distinct enzymes. mmBCFA biosynthesis 
uses specific enzymes for some reactions. In $C$. elegans, for instance, ELO-3 condenses straight chain- and FAs, and ELO-5 and ELO-6 condense branched-chain FAs, whereas LET-767 is used for the reduction of either. Middle Right: Biosynthesis of unsaturated FAs. Saturated FAs are desaturated to monounsaturated and polyunsaturated FAs (MUFAs and PUFAs) by a wide range of desaturase-(delta 5-, 6-, 9, 12 and omega-3) and PUFA elongase-(omega-3 and 6) activities to generate a variety of unsaturated FAs with different lengths and different numbers of double bonds. In contrast to higher plants and mammals, $C$. elegans possesses all of the enzymes required for the de novo biosynthesis of 20:4(n-6) and 20:5(n-3) FAs. Starting with the primer acetyl-CoA, the biosynthesis of the PUFA 20:5(n-3) by this route involves $\sim 30$ distinct enzymes and 70 reactions. Bottom: Complex lipids. Products serve as building blocks for complex lipids, with saturated FAs dominating sphingolipids (SLs) and desaturated FAs dominating glycerols and phospholipids.

Corresponding metabolic enzyme genes with experimentally confirmed functions in $C$. elegans are indicated in red. 
a

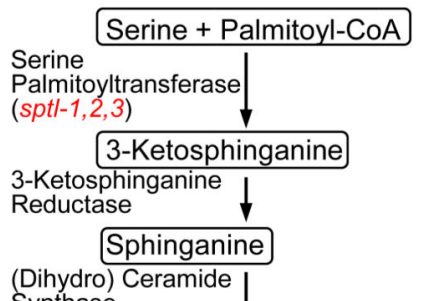

\section{A A}

b

Synthase
(lagr-1, hyl-1,2)

Dihydroceramide

(asm-1,2,3)

Dihydrosphingomyelin

Sphingolipid-fatty Acid

Hydroxylase? (fath-1)
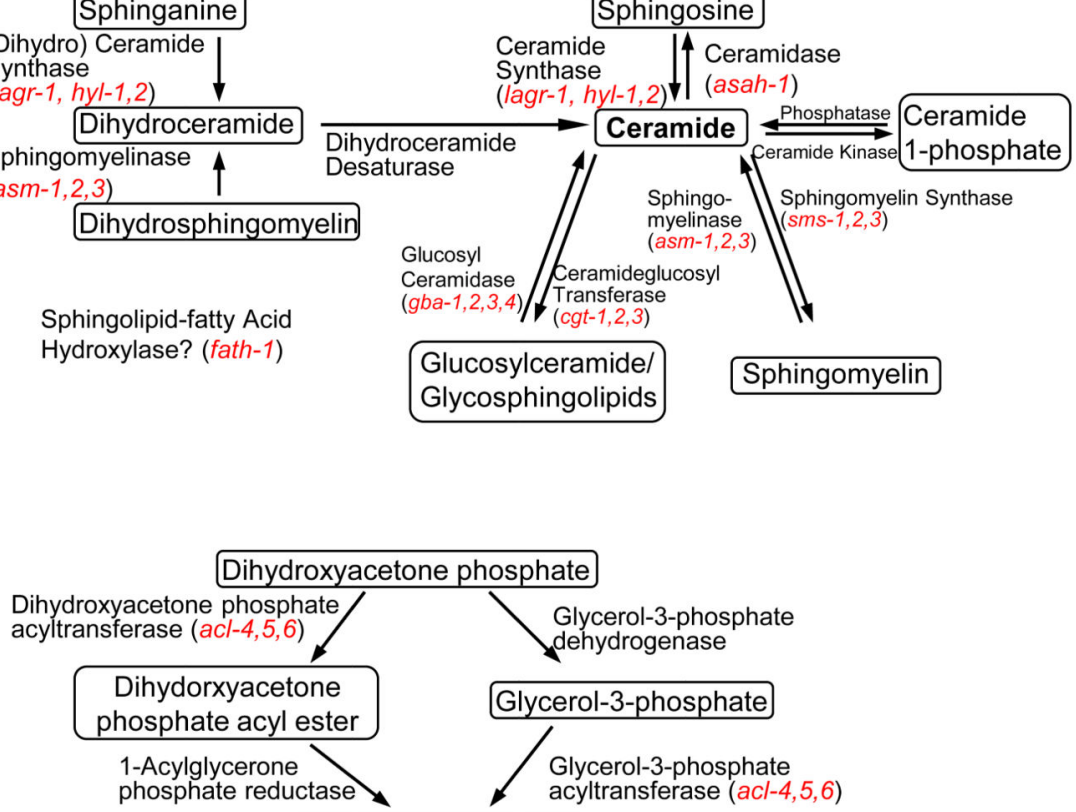

Lysophosphatidic acid

Lysophosphatidate
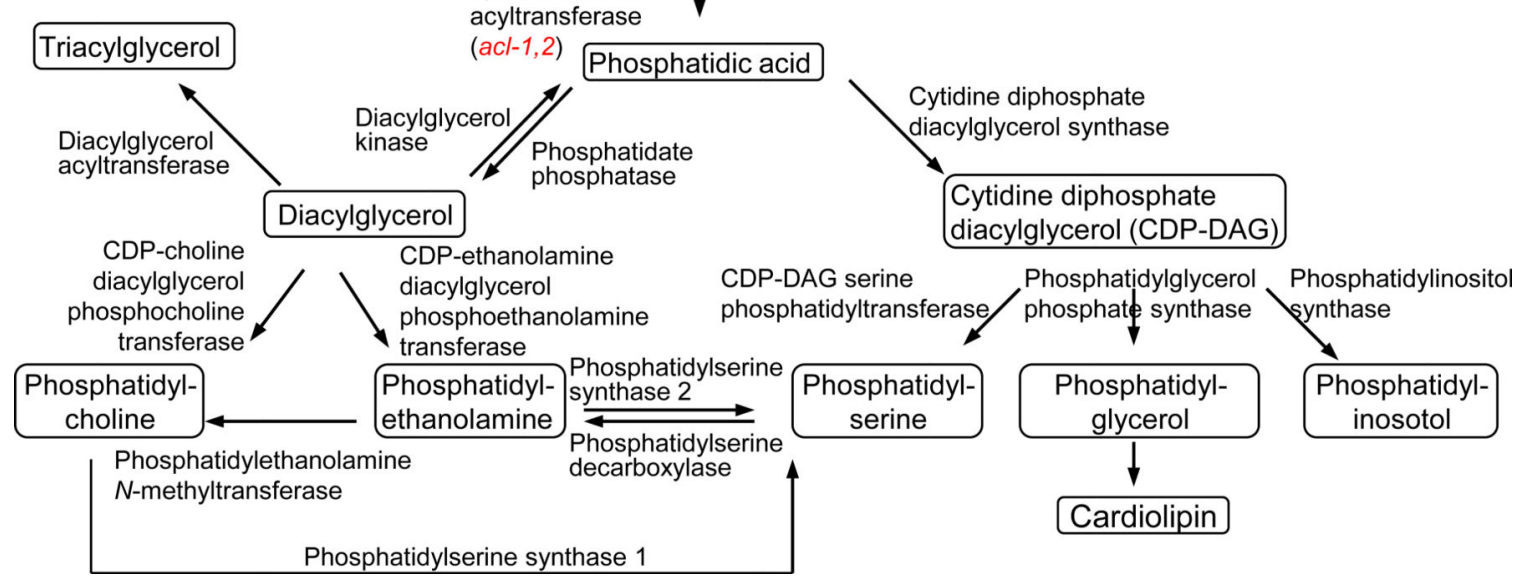

Figure 2. Conserved sphingolipid and phospholipid metabolic pathways. (a) Sphingolipid metabolic pathways

Left: De novo biosynthesis. The biosynthesis of sphginolipids (SLs) begins with the condensation of serine and palmitoyl-CoA. Three further synthetic steps are needed to produce ceramide (Cer) that links the sphingoid backbone (long chain base) with a FA chain and is the center of the SL metabolic pathway. Right: Salvage pathways and complex SL

biosynthesis. The post-Cer metabolic steps are reversible. Ceramide serves both as substrate and as product for the biosynthesis and degration, respectively, of complex SLs, such as sphingomyelin or glycosphingolipids. Deacylation of Cer yields sphingosine that can be 
phosphorylated to sphingosine-1-phosphate. The irreversible degradation of the sphingoid backbone is catalyzed by a lyase that acts on sphingosine-1-phosphate. (b) Major

glycerophospholipid metabolic pathways. Top: Biosynthesis of phosphatidic acid, which is an intermediate in the biosynthesis of phospholipids, by a series of reduction and acylation reactions. Bottom Left: the Kennedy pathway. Phosphatidic acid is dephosphorylated to yield diacylglycerol, which in turn is converted into phosphatidylcholine (PC) or phosphatidylethanolamine (PE) by the addition of a phosphocholine or a phosphoethanolamine head group, respectively. Phosphatidylserine (PS) can be formed by exchanging the head group from either PC or PE for serine. Mitochondrial PE is synthesized on location by decarboxylation of PS. Bottom Right: Cytidine diphosphate diacylglycerol (CDP-DAG) pathway. PS and phosphatidylinositol (PI) are synthesized from the precursor CDP-DAG by the enzymes CDP-DAG serine phosphatidyltransferase and CDP-DAG inositol phosphatidyltransferase (PI synthase), respectively. CDP-DAG is also used for the synthesis of phosphatidylglycerol and cardiolipin, which are phospholipids that are confined to mitochondrial membranes. Corresponding metabolic enzyme genes with experimentally confirmed functions in C. elegans are indicated in red. 


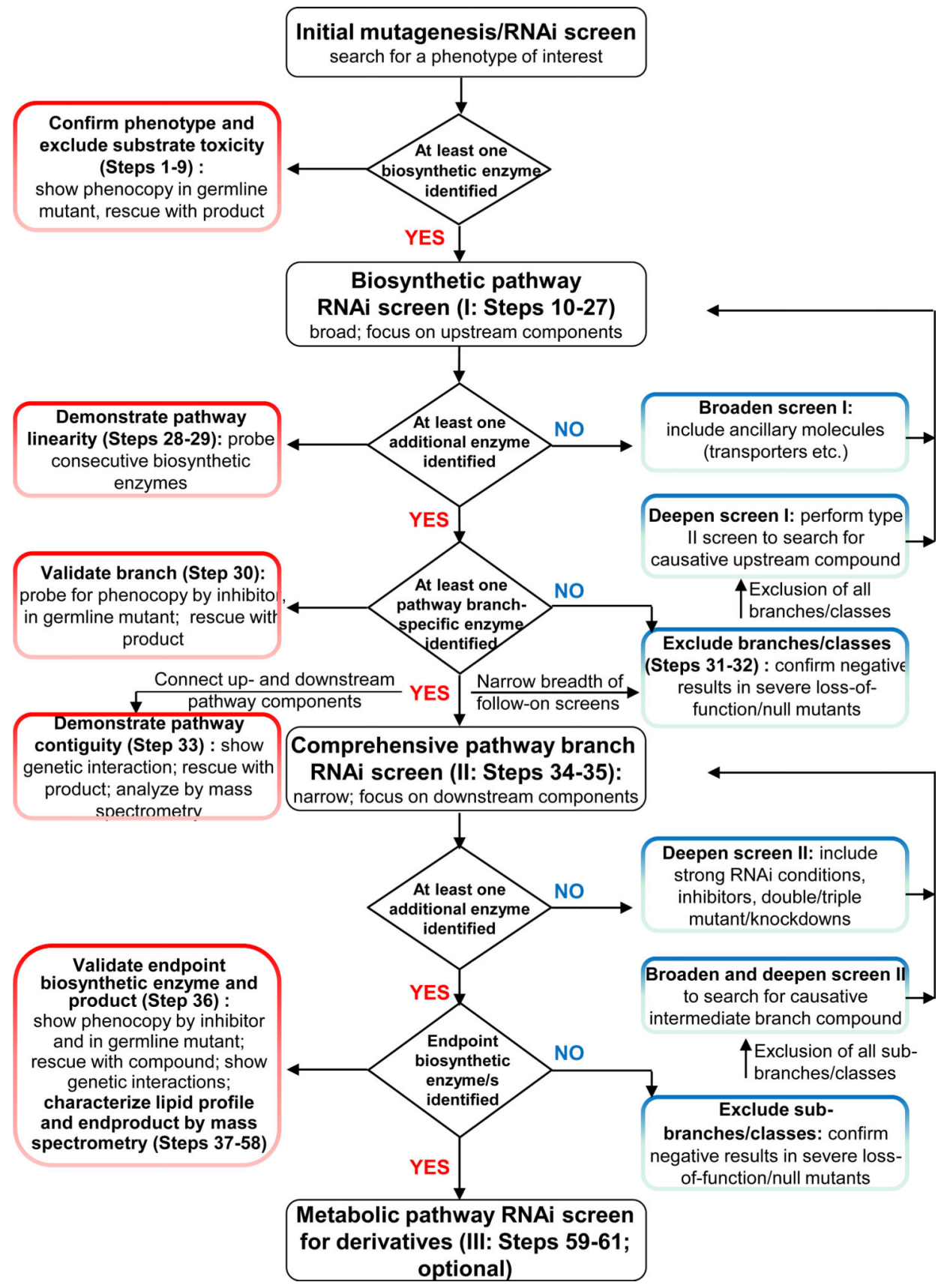

Figure 3. Work flow chart for a tiered lipid biosynthetic pathway RNAi screen

Top to bottom: RNAi screens become successively constricted, zooming in onto the endpoint biosynthetic enzyme to identify the underlying common lipid compound. The broad initial pathway screen (I; Steps 10-27) aims to identify the underlying metabolite category/class and pathway branch and, typically, has a focus on simple metabolites. The second stage in-depth screen of an identified pathway branch (II; Steps 34 and 35) aims to identify the specific lipid end product and, depending on the identified enzymes, is focused on complex metabolites. An additional screen for derivatives of the identified end product (III; Steps 59-61) can be performed based on the same principles, and has the potential to 
identify its exact chemical characteristics. Positive hits (phenocopy; left column) confirm initial findings, identify roles for intermediates, define components of the searched-for end product and guide the screen towards the downstream endpoint biosynthetic enzyme. Negative results (no phenocopy; right column), confirmed under severe loss-of-function conditions (e.g., nulls), exclude pathway arms and thus multiple compounds and narrow down follow-on screens, allowing in-depth screening that includes the simultaneous depletion of paralogs and a broader range of depletion conditions. If no downstream enzymes are identfied, they redirect screens to repeat screening of a broader selection of molecules and/or screening with more severe depletion conditions, to the search for the causative compound among upstream components of the pathway. 
Table 1

Critical C. elegans fatty acid biosynthetic enzymes

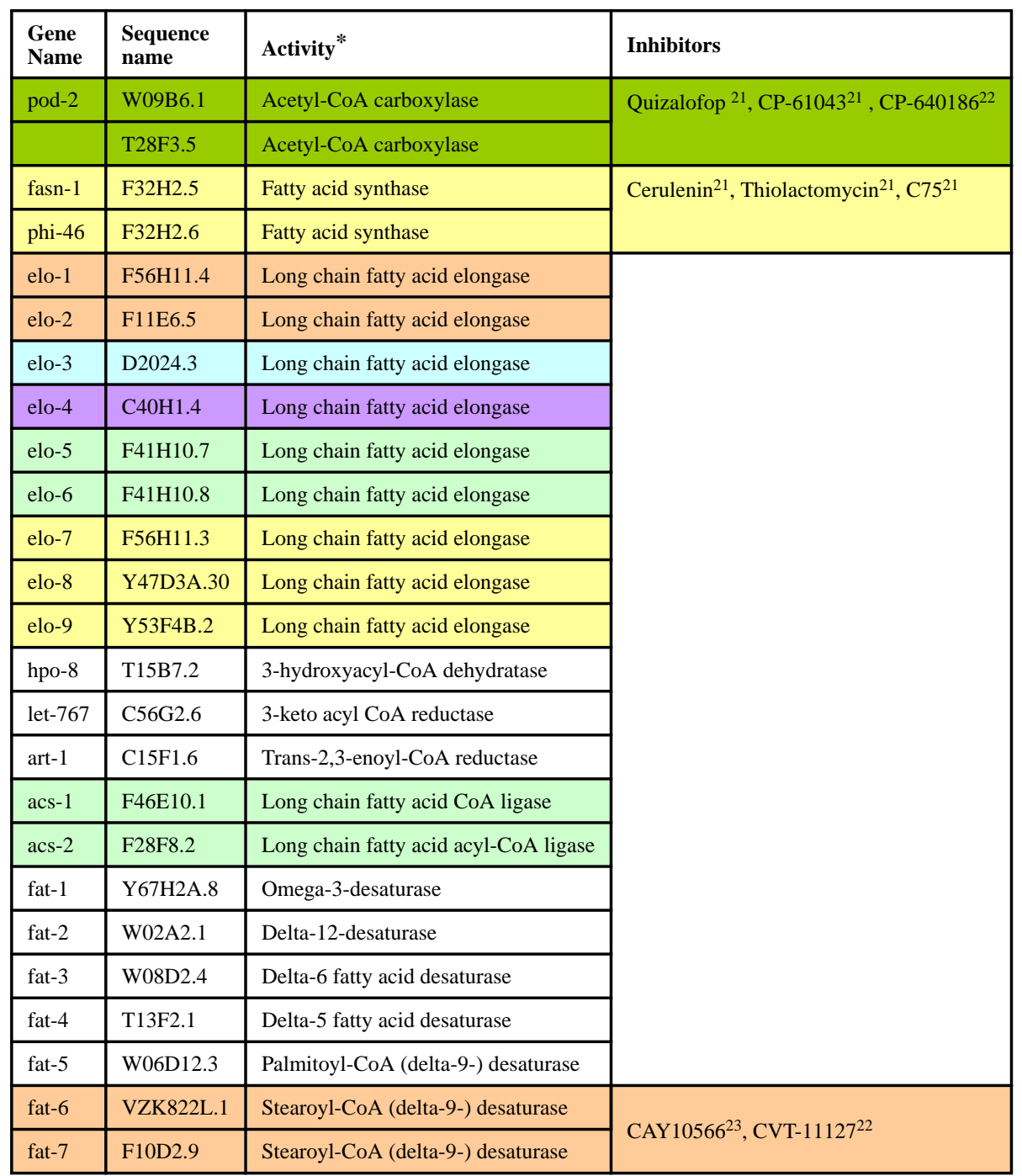

List is not comprehensive but includes critical biosynthetic reactions and closely related family members/paralogs (grouped by color) of biosynthetic enzymes. Genes with unknown names are left blank

http://www.wormbase.org 


\section{Table 2}

Critical C. elegans sphingolipid metabolic enzymes and inhibitors

\begin{tabular}{|c|c|c|c|}
\hline $\begin{array}{l}\text { Gene } \\
\text { name }\end{array}$ & $\begin{array}{l}\text { Sequence } \\
\text { name }\end{array}$ & Activity $^{*}$ & Inhibitors $^{24,25}$ \\
\hline sptl-1 & $\mathrm{C} 23 \mathrm{H} 3.4$ & Serine palmitoyltransferase & \multirow{3}{*}{$\begin{array}{l}\text { Myriocin and its derivatives, sphingofungin B \& C, cycloserine, beta- } \\
\text { choroalanine }\end{array}$} \\
\hline sptl-2 & F43H9.2 & Serine palmitoyltransferase & \\
\hline \multirow[t]{5}{*}{ sptl-3 } & T22G5.5 & Serine palmitoyltransferase & \\
\hline & Y37E11AM.3 & 3-Ketosphinganine reductase & \\
\hline & Y54E5A.1 & Dihydroceramide desaturase & \multirow{2}{*}{ GT11 and analogues, Dihydroceramide analogues } \\
\hline & F33D4.4 & Dihydroceramide desaturase & \\
\hline & $\mathrm{C} 25 \mathrm{~A} 1.5$ & \multicolumn{2}{|l|}{ Sphingolipid fatty acid hydroxylase } \\
\hline hyl-1 & C09G4.1 & Ceramide synthase & \multirow{3}{*}{ Fumonisin and related compounds } \\
\hline hyl-2 & K02G10.6 & Ceramide synthase & \\
\hline lagr-1 & Y6B3B.10 & Ceramide synthase & \\
\hline \multirow[t]{4}{*}{ asah-1 } & $\mathrm{K} 11 \mathrm{D} 2.2$ & Acid ceramidase & \multirow{3}{*}{ Alpha-ketoamides, Ceramide analogues, Desipramine } \\
\hline & F27E5.1 & Acid ceramidase & \\
\hline & Y55D5A.3 & Acid ceramidase & \\
\hline & W02F12.2 & \multicolumn{2}{|l|}{ Alkaline ceramidase } \\
\hline cgt-1 & $\mathrm{T} 06 \mathrm{C} 12.10$ & Ceramide glucosyltransferase & \multirow{3}{*}{ d,l-PDMP, D-DPMA, D-threo-PPPP, N-butyldeoxynojirimycin, OGT2378 } \\
\hline cgt-2 & F20B4.6 & Ceramide glucosyltransferase & \\
\hline cgt-3 & F59G1.1 & Ceramide glucosyltransferase & \\
\hline gba-1 & $\mathrm{C} 33 \mathrm{C} 12.3$ & Beta-glucocerebrosidase & \multirow{4}{*}{ D-gluconolactone, conduritol B epoxide, cyclophellitol, conduritol B aziridine } \\
\hline gba-2 & $\mathrm{C} 33 \mathrm{C} 12.8$ & Beta-glucocerebrosidase & \\
\hline gba-3 & F11E6.1 & Beta-glucocerebrosidase & \\
\hline \multirow[t]{2}{*}{ gba-4 } & Y4C6B.6 & Beta-glucocerebrosidase & \\
\hline & $\mathrm{C} 29 \mathrm{E} 4.10$ & Orthologous to human galactocerebrosidase & \\
\hline sms-1 & H21P03.3 & Sphingomyelin synthase & \multirow{3}{*}{ MS-209, D609 } \\
\hline sms-2 & F53H8.4 & Sphingomyelin synthase & \\
\hline \multirow[t]{3}{*}{ sms-3 } & Y22D7AL.8 & Sphingomyelin synthase & \\
\hline & F53B1.2 & Sphingomyelin synthase-related protein & \\
\hline & W07E6.3 & Sphingomyelin synthase-like domain-containing & \\
\hline asm-1 & B0252.2 & Acid sphingomyelinase & \multirow{3}{*}{ SR33557, Desipramine, GW4869, Scyphostatin, Manumycin A, SM analogues } \\
\hline asm-2 & ZK455.4 & Acid sphingomyelinase & \\
\hline \multirow[t]{3}{*}{ asm-3 } & W03G1.7 & Acid sphingomyelinase & \\
\hline & ZK856.5 & asm-like phosphodiesterase & \\
\hline & T27F6.6 & Sphingomyelinase family protein & \\
\hline \multirow[t]{2}{*}{ sphk-1 } & C34C6.5 & Sphingosine kinase & $\begin{array}{l}\text { N,N-dimethylsphingosine, LCL204, L-threo-sphinganine, SG12, SG14, SKi, } \\
\text { phenoxodiol }\end{array}$ \\
\hline & T10B11.2 & Ceramide kinase & F-12509A \\
\hline spl-1 & Y66H1B.4 & Sphingosine phosphate lyase & \multirow{2}{*}{ FTY720, 1-desoxysphinganine-1-phosphonate, 2-vinylsphinganine-1-phosphate } \\
\hline $\operatorname{tag}-38$ & B0222.4 & Sphingosine phosphate lyase & \\
\hline
\end{tabular}




\begin{tabular}{|l|l|l|l|}
\hline $\begin{array}{l}\text { Gene } \\
\text { name }\end{array}$ & $\begin{array}{l}\text { Sequence } \\
\text { name }\end{array}$ & Activity $^{*}$ & Inhibitors $^{24,25}$ \\
\hline & Y104H12D.3 & Sphingosine phosphate lyase & \\
\hline
\end{tabular}

List is not comprehensive but includes critical biosynthetic reactions and closely related family members/paralogs (grouped by color) of metabolic enzymes. Genes with unknown names are left blank.

http://www.wormbase.org 


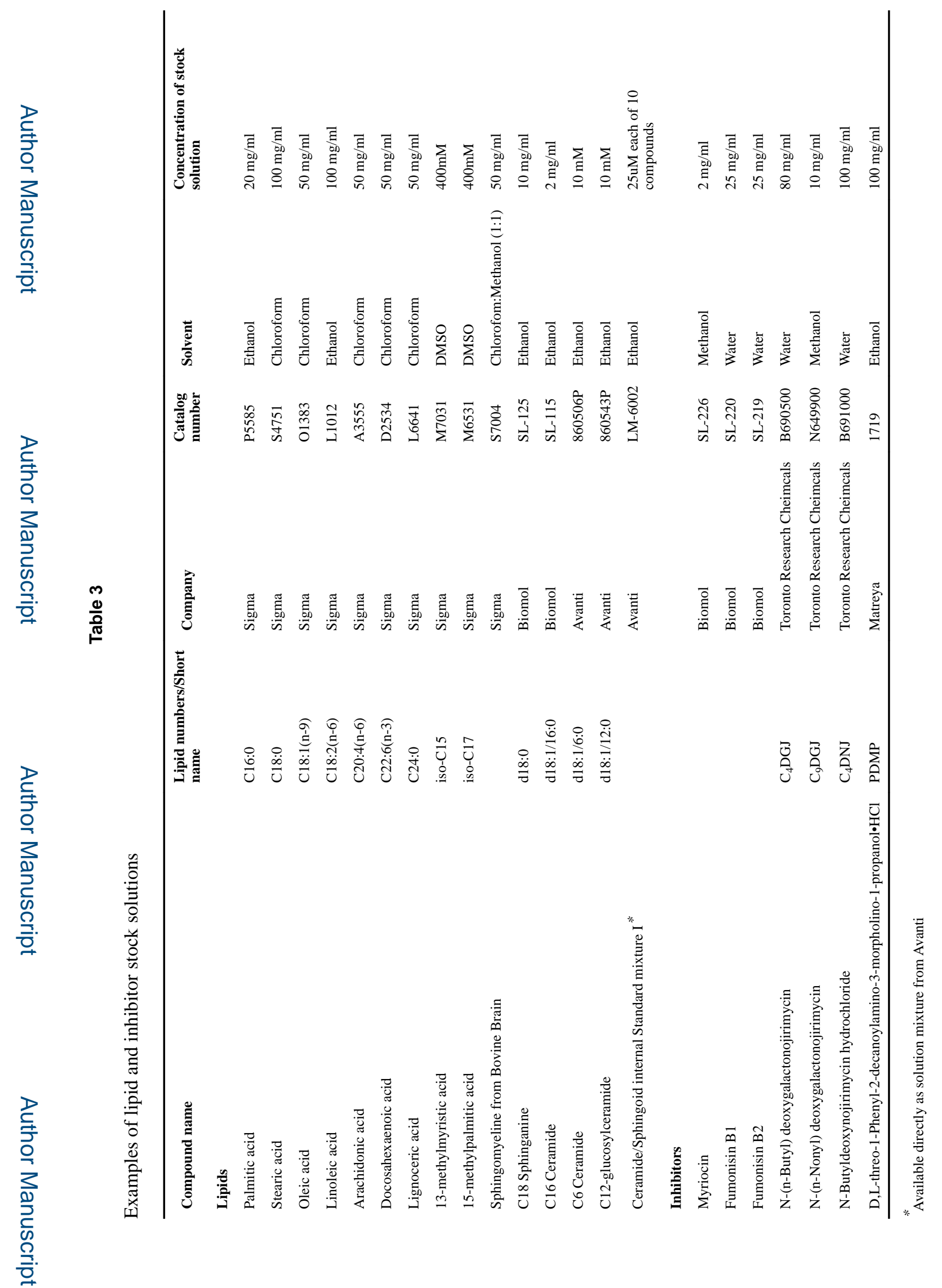

Nat Protoc. Author manuscript; available in PMC 2017 September 13. 
Table 4

Troubleshooting table

\begin{tabular}{|c|c|c|c|}
\hline Steps & Problem & Possible reason & Solution \\
\hline \multirow[t]{6}{*}{$5-9$} & $\begin{array}{l}\text { Exogenous lipid supplementation } \\
\text { does not rescue the phenotype }\end{array}$ & The phenotype is too severe & $\begin{array}{l}\text { Use mild RNAi conditions or a mild reduction-of- } \\
\text { function mutant }\end{array}$ \\
\hline & & $\begin{array}{l}\text { The lipid species used for rescue } \\
\text { resides upstream of the identified } \\
\text { enzyme }\end{array}$ & $\begin{array}{l}\text { Choose a lipid species downstream of the identified } \\
\text { enzyme, e.g. its direct product }\end{array}$ \\
\hline & & Amount too low & Increase the final lipid concentration on the plate \\
\hline & & $\begin{array}{l}\text { Lipid uptake is poor(can test by } \\
\text { using labeled lipids) }\end{array}$ & $\begin{array}{l}\text { Can try microinjection or mix lipids into the agar in the } \\
\text { presence of tergitol }^{32}\end{array}$ \\
\hline & & $\begin{array}{l}\text { Supplied lipids do not have } \\
\text { organism-specific structure }\end{array}$ & $\begin{array}{l}\text { May require lipid extraction from the animal and feed } \\
\text { back }\end{array}$ \\
\hline & & $\begin{array}{l}\text { Lipid biosynthetic process itself } \\
\text { is linked to function }\end{array}$ & $\begin{array}{l}\text { Overexpress the corresponding lipid biosynthetic } \\
\text { enzyme; an alternative approach to prove pathway } \\
\text { contiguity and/or the link between lipid and cellular } \\
\text { process may be required }\end{array}$ \\
\hline \multirow[t]{5}{*}{27} & $\begin{array}{l}\text { Phenotype not consistently present } \\
\text { in animals depleted of enzymes in } \\
\text { a linear stretch of the pathway (or } \\
\text { absence of phenotype in any other } \\
\text { unexpected scenario) }\end{array}$ & $\begin{array}{l}\text { The specific transcripts are RNAi } \\
\text { resistant }\end{array}$ & Examine the corresponding germline mutant \\
\hline & & RNAi too mild & Use RNAi sensitive strains \\
\hline & & Existence of maternal effect & Score phenotype of the third generation \\
\hline & & $\begin{array}{l}\text { The gene functions redundantly } \\
\text { with other genes }\end{array}$ & $\begin{array}{l}\text { Knock down paralogs/redundant genes by double/triple } \\
\text { RNAi/mutant analysis }\end{array}$ \\
\hline & & $\begin{array}{l}\text { Phenotype is masked by sterility } \\
\text { or early embryonic lethality }\end{array}$ & $\begin{array}{l}\text { Mild, post-embryonic or tissue-specific RNAi can be } \\
\text { tried. Mild RNAi conditions can be empirically } \\
\text { determined for each clone by titrating IPTG } \\
\text { concentrations over a wide range of concentrations and } \\
\text { by diluting RNAi bacteria with control RNAi bacteria. } \\
\text { RNAi can be initiated at any stage after completion of } \\
\text { embryogenesis by placing eggs or larvae or adults on } \\
\text { RNAi plates for evaluating the same generation. RNAi } \\
\text { can be performed in a tissue-specific RNAi strain. }\end{array}$ \\
\hline 29 & $\begin{array}{l}\text { Depletion of any additional } \\
\text { downstream lipid biosynthetic } \\
\text { enzyme by any method available } \\
\text { produces no phenotype }\end{array}$ & $\begin{array}{l}\text { The lipid product of the furthest } \\
\text { downstream identified lipid } \\
\text { biosynthetic enzyme is the } \\
\text { searched-for compound }\end{array}$ & $\begin{array}{l}\text { This can be demonstrated by rescue of the phenotype in } \\
\text { a double mutant/RNAi animal of the corresponding } \\
\text { upstream and downstream biosynthetic enzymes with } \\
\text { this lipid compound }\end{array}$ \\
\hline \multirow[t]{2}{*}{ 30(B)(iv) } & $\begin{array}{l}\text { The small-molecule inhibitor fails } \\
\text { to copy the phenotyperesulting } \\
\text { from the loss of the corresponding } \\
\text { biosynthetic enzyme }\end{array}$ & $\begin{array}{l}\text { Inhibitor concentration or uptake } \\
\text { too low }\end{array}$ & Increase concentration; try microinjection \\
\hline & $\begin{array}{l}\text { The range of effective dose of } \\
\text { inhibitor is narrow (no effect with } \\
\text { lower, and early lethality with } \\
\text { higher, dose) }\end{array}$ & $\begin{array}{l}\text { The inhibitor has weak potency } \\
\text { and lacks specificity }\end{array}$ & $\begin{array}{l}\text { Can attempt to carefully titrate inhibitor concentration; } \\
\text { check ability of the inhibitor to enhance the (mild) loss } \\
\text { of the corresponding biosynthetic enzyme }\end{array}$ \\
\hline 33A(ii) & $\begin{array}{l}\text { The expected enhancement } \\
\text { between incompleteloss-of- } \\
\text { function conditionsof two enzymes } \\
\text { is not detected }\end{array}$ & Phenotype(s) is too strong & $\begin{array}{l}\text { Try different combinations of mutant alleles and RNAi } \\
\text { conditions }\end{array}$ \\
\hline
\end{tabular}

